\title{
Modelling Macrophage Infiltration into Avascular Tumours
}

\author{
C.E. KELLY ${ }^{\mathrm{a}}$, R.D. LEEK ${ }^{\mathrm{b}}$, H.M. BYRNE ${ }^{\mathrm{a} *}$, S.M. COX ${ }^{\mathrm{a}}$, A.L. HARRIS ${ }^{\mathrm{b}}$ and C.E. LEWIS \\ ${ }^{\mathrm{a}}$ School of Mathematical Sciences, University of Nottingham, Nottingham NG7 2RD, UK; ${ }^{\mathrm{b}}$ ICRF Molecular Oncology Laboratory, University of Oxford, \\ Institute of Molecular Medicine, John Radcliffe Hospital, Oxford OX3 9DU, UK; ${ }^{\mathrm{D}}$ Department of Pathology, The University of Sheffield Medical School, \\ Sheffield S10 2JF, UK
}

(Received 5 August 2000; In final form 23 April 2001)

\begin{abstract}
In this paper a mathematical model that describes macrophage infiltration into avascular tumours is presented. The qualitative accuracy of the model is assessed by comparing numerical results with independent experimental data that describe the infiltration of macrophages into two types of spheroids: chemoattractant-producing (hepa-1) and chemoattractant-deficient (or C4) spheroids. A combination of analytical and numerical techniques are used to show how the infiltration pattern depends on the motility mechanisms involved (i.e. random motion and chemotaxis) and to explain the observed differences in macrophage infiltration into the hepa-1 and $\mathrm{C} 4$ spheroids. Model predictions are generated to show how the spheroid's size and spatial structure and the ability of its constituent cells influence macrophage infiltration. For example, chemoattractant-producing spheroids are shown to recruit larger numbers of macrophages than chemoattractant-deficient spheroids of the same size and spatial structure. The biological implications of these results are also discussed briefly.
\end{abstract}

\section{INTRODUCTION}

Solid tumours consist of many different cell types, not only malignant cells but also such cells as lymphocytes, fibroblasts, endothelial cells and macrophages (Lewis et al., 1995). Initially, the presence of macrophages, a type of white blood cell, within solid tumours was considered a good prognostic sign as they were thought to migrate into the tumour from the blood and lyse/phagocytose tumour cells as part of the host's immune response to the "foreign growing body" (Mantovani et al., 1992). This description is consistent with the usual role of macrophages as part of the immune system's response to injury, infection and inflammation. While there is limited evidence indicating that the presence of large numbers of macrophages within a given tumour type is beneficial to the patient (Pupa et al., 1996), compelling results are now being published which show the function of macrophages within tumours to be more complex than this. For example, high levels of macrophage infiltration are associated with good prognosis in colorectal cancer (Toomey et al., 1999) but poor prognosis in breast cancer (Leek et al., 1996). Such diametrically opposed conclusions indicate that macrophages possess a large repertoire of functions, with different tumours activating macrophages to perform different combinations of pro- and anti-tumour functions (Elgert et al., 1998).

Tumour-inhibitory functions that macrophages are known to perform include tumour cell lysis, a process in which the macrophages bind to tumour cells and secrete a cytotoxic substance directly into the target cell, and such cytokines as interleukins 1 and 6 , and tumour necrosis factor alpha which retard growth (Elgert et al., 1998). Equally, macrophages may indirectly promote a tumour's development and survival by secreting other cytokines such as interleukin 10, prostaglandins and transforming growth factor-beta 1 (TGF- $\beta$ ) which suppress anti-tumour immune responses (Elgert et al., 1998). The ability of macrophages to promote tumour angiogenesis is perhaps their most important, pro-tumour function. Indeed, we have recently demonstrated a positive correlation between macrophage numbers and tumour vascularity in breast tumours (Leek et al., 1996). This effect of macrophages is achieved through the release of tissue-matrix degrading proteases and cytokines, which attract and stimulate the growth of endothelial cells such as vascular endothelial growth factor (VEGF) (reviewed by Lewis et al. (1999)). In summary, as Fig. 1 shows, the role of macrophages in regulating tumour growth and progression can be viewed as a balance between anti-tumour and pro-tumour functions.

*Corresponding author. 


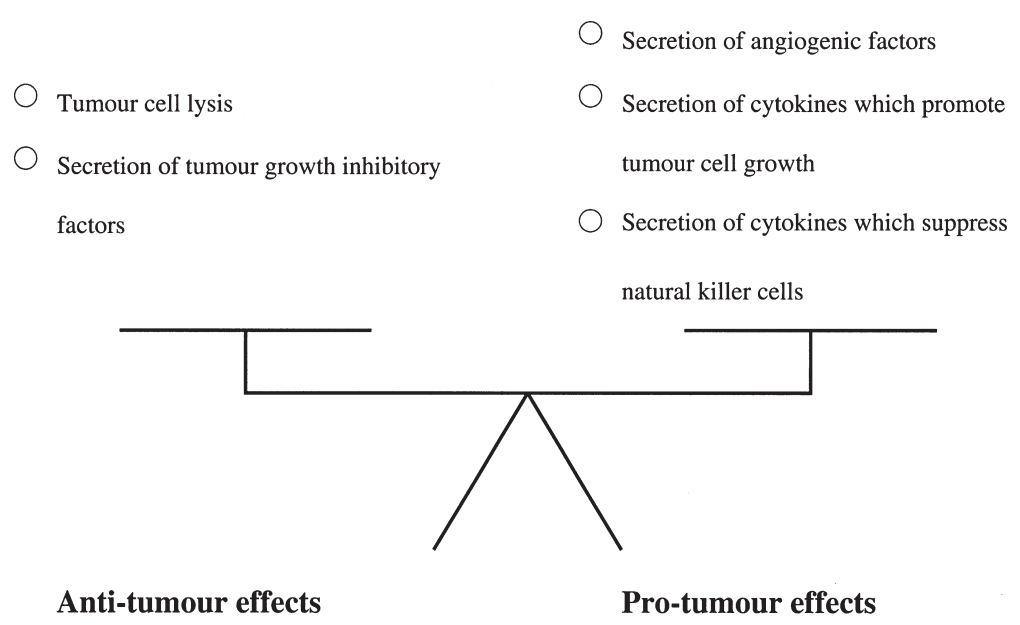

FIGURE 1 The resultant effect of macrophages on a tumour may be viewed as a balance between anti- and pro-tumour effects. Anti-tumour functions include tumour cell kill through the expression of cytotoxic chemicals and growth inhibitory factors. Pro-tumour functions include the expression of growth promoting factors and angiogenic factors.

Our recent data show that macrophages congregate specifically in regions of breast tumours where there is poor vascularity and hence a reduced supply of vital nutrients such as oxygen and glucose (Leek et al., 1996). Tumour cells proliferate only slowly in these hypoxic areas. This pattern of macrophage migration may be due, in part, to hypoxia (low levels of oxygen) inducing tumour cells to secrete chemoattractants for macrophages such as VEGF, interleukin 8 and TGF- $\beta$ (Santilli et al., 1991; Shi et al., 1999; Leek et al., 2000; Lewis et al., 2001). Chemotaxis would draw macrophages into hypoxic sites as they migrate up a gradient of such cytokines. However, there is also some evidence for macrophages becoming immobilised once they reach hypoxic areas. Monocyte chemoattractant protein-1 (MCP-1) is upregulated in many forms of tumour and its expression correlated with macrophage infiltration in ovarian cancer (Negus et al., 1997).

However, expression of MCP-1 by ovarian cancer cells is down-regulated by hypoxia, suggesting that, once macrophages localise to hypoxic areas, they may not be able to migrate out again, i.e. that the MCP-1 trail they were following has "gone cold" (Negus et al., 1998). Furthermore, a cytokine known as macrophage migration inhibitory factor (MIF) is also expressed more abundantly by hypoxic than normoxic tumour cells (Koong et al., 2000). As its name suggests, MIF inhibits the migration of macrophage cells, so its over-expression in hypoxic areas may help to immobilise macrophages in hypoxic areas. At present, the relative contribution made by hypoxicinduced chemoattractants and immobilising cytokines to the marked accumulation of macrophages in hypoxic areas of tumours is not known.

However, it is clear that, once they reach hypoxic areas, exposure to low oxygen levels stimulates macrophages to secrete such pro-angiogenic factors as VEGF and TNF- $\alpha$ (Lewis et al., 1999; Lewis et al., 2001). These, in turn, stimulate ingrowth of vessels and the re-oxygenation of the tumour cells in the hypoxic areas. This enables them to recommence growing at their usual, rapid pace. The new blood vessels also provide an escape route for the tumour cells into the general circulation. Thus, macrophage accumulation in hypoxic areas is important for the ongoing growth and spread of human tumours.

Hypoxic tumour cells have, to date, proven notoriously difficult to treat with standard anti-cancer therapies: there are problems with drug delivery (hypoxic regions are usually at the furthest point from any perfusing blood vessels) and chemotherapeutic drugs are largely ineffective in hypoxic areas as they are designed to kill proliferating cells. We have, therefore, designed a new, gene-based therapy, which exploits the tendency of macrophages to migrate into hypoxic tumour regions to target expression of therapeutic genes to these areas. In this new therapy, a cancer patient's macrophages are removed and a therapeutic gene inserted that is only activated under hypoxia. When the cells are re-injected back into the patient's bloodstream, they extravasate, as usual, into the tumour, migrate into the hypoxic areas and then release the protein encoded by the therapeutic gene. These proteins can be cytotoxic chemicals that kill neighbouring tumour cells or anti-angiogenic factors that retard, or halt, the ingrowth of blood vessels (Griffiths et al., 2000). It is hoped that macrophages can be used in this way to target gene expression to hypoxic areas of both large, well-established primary tumours and their small, avascular metastatic deposits around the body.

To help optimise the use of genetically engineered macrophages to target hypoxic sites within early (small) avascular tumours, we present in this paper a mathematical model that describes macrophage infiltration into avascular tumour spheroids (three-dimensional tumour masses grown in vitro). The model is compared with our independent, biological data in which the infiltration patterns of macrophages into two types of tumour spheroids were studied (Leek, 1999). In this model, the tumour-infiltrating macrophages are viewed as "passive", in the sense that they perform neither pro- nor anti-tumour 
functions (the inclusion of such effects is the subject of work in progress). Owen and Sherratt have developed complementary mathematical models that focus on antitumour macrophage functions such as lysis (Owen and Sherratt, 1997; Owen and Sherratt, 1998; Owen and Sherratt, 1999). For example, they developed a spatially uniform model to describe the combined effects of macrophage aggregation and cytolysis on tumour growth (Owen and Sherratt, 1998; Owen and Sherratt, 1999). By analysing their model, they concluded that a cytolytic response to tumours, initiated by the immune response, would not be effective at eliminating a tumour. However they suggested that the addition to the tumour of chemicals that stimulate macrophage infiltration might lead to tumour elimination. By extending their original model to include spatial effects (e.g. random motion) and carrying out a linearised stability analysis, Owen and Sherratt went on to suggest that the presence of macrophages within tumours may lead to the generation of spatio-temporal heterogeneity (Owen and Sherratt, 1997). A weakness of Owen and Sherratt's models is their failure to take account of the tumour's spatial structure (with regions of hypoxia and necrosis adjacent to regions of high cell proliferation), and the importance of chemotaxis as a mechanism for macrophage motion. These features are included in the model that we present in this paper.

Macrophages play an important role in many pathological processes other than cancer and in various stages or embryonic development (Gordon, 1995). For example, they have been implicated in atherosclerosis (Babaev et al., 1999), rheumatoid arthritis (Mapp et al., 1995; Bischof et al., 2000), HIV infection (Wodarz et al., 1998), lung disease (Tran et al., 1999a, b) and tissue remodelling in wound healing (Bennet and Schulz, 1993) and digit development (Dupe et al., 1999). Their involvement in such a diverse array of tissues is undoubtedly related to the vast array of functions that macrophages may perform, the precise function depending on their activation status and environmental conditions. Faced with such a complex cell, the prospect of developing mathematical models that will provide physical insight into the role that macrophages play in human biology may appear infeasible. However, upon closer inspection, it is possible to see that these different processes have several features in common, and that these could be exploited when developing mathematical models. For example, the chemotactic migration of macrophages to hypoxic sites at which they are activated to express angiogenic factors is characteristic of tumours, wound healing and rheumatoid arthritis (reviewed by (Lewis et al., 1999)). In summary, it is highly likely that the model presented in this paper could be adapted or extended to study other processes (e.g. wound healing, arthritis and atherosclerosis) in which macrophage function is important.

The outline of the paper is as follows. In "The experimental results" we present Leek's experimental results (Leek, 1999), before developing our mathematical model in "The mathematical model" section. "The numerical results" and "Mathematical analysis of the model" sections contain numerical and analytical results. The paper concludes in "Discussion" with a summary of the paper and a brief discussion of ways in which the model could be extended.

\section{THE EXPERIMENTAL RESULTS}

Analysis of biopsies taken from cancer patients shows that tumour associated macrophages (TAMs) localise in hypoxic regions, away from blood vessels. Additionally, there is evidence to suggest that macrophage infiltration may be correlated with tumour necrosis. In order to determine whether TAMs are attracted into hypoxic regions by products generated from neighbouring, necrotic regions or whether they are actively recruited by chemicals such as hif-1 which are upregulated under hypoxia, Leek (1999) studied macrophage infiltration into avascular spheroids cultured from hepa-1 and C4 cells, two cell lines which differ in their ability to express hif-1, a protein which is known to upregulate the production of VEGF under hypoxia. Since VEGF is a potent macrophage chemoattractant, it is anticipated that hepa-1 spheroids, which express hif-1, will produce macrophage chemoattractants under hypoxia whereas the hif-l-deficient $\mathrm{C} 4$ spheroids will not. Thus any observed differences in the macrophage infiltration pattern between the two cell lines may be ascribed to the presence or absence of macrophage chemoattractants. To assess the degree of macrophage infiltration into the spheroids, they were stained with an antibody. A computerised image analyser was used to measure the digitised image and the results for each experiment expressed in fluorescent object units.

The first set of experiments carried out by Leek (1999) followed the infiltration of macrophages into 10-day old hepa- 1 and $\mathrm{C} 4$ spheroids over a $12 \mathrm{~h}$ period, 10-day old spheroids being used since they possess regions of necrosis and hypoxia. Figure $2 \mathrm{a}-\mathrm{d}$ show the results after 2, 4, 6 and $12 \mathrm{~h}$ co-culture, respectively. In both the hepa-1 and $\mathrm{C} 4$ tumour cell lines the number of macrophages contained within the spheroids increases steadily over time. In addition, the hepa-1 spheroids are initially more successful than the $\mathrm{C} 4$ spheroids at recruiting macrophages, an observation which is consistent with the hypothesis that, unlike the hepa-1 spheroids, the $\mathrm{C} 4$ spheroids do not express macrophage chemoattractants. After $12 \mathrm{~h}$ co-culture, the situation reverses, so that, eventually, the $\mathrm{C} 4$ spheroids accumulate larger numbers of macrophages than their hepa- 1 counterparts.

The second set of experiments carried out by Leek (1999) was designed to investigate the effect of tumour size and composition on macrophage infiltration. The distribution of macrophages within 5, 10 and 20-day old spheroids after $6 \mathrm{~h}$ co-culture was recorded and the results are presented in Fig. 3. The macrophage profiles are qualitatively similar in all cases, with the peak 

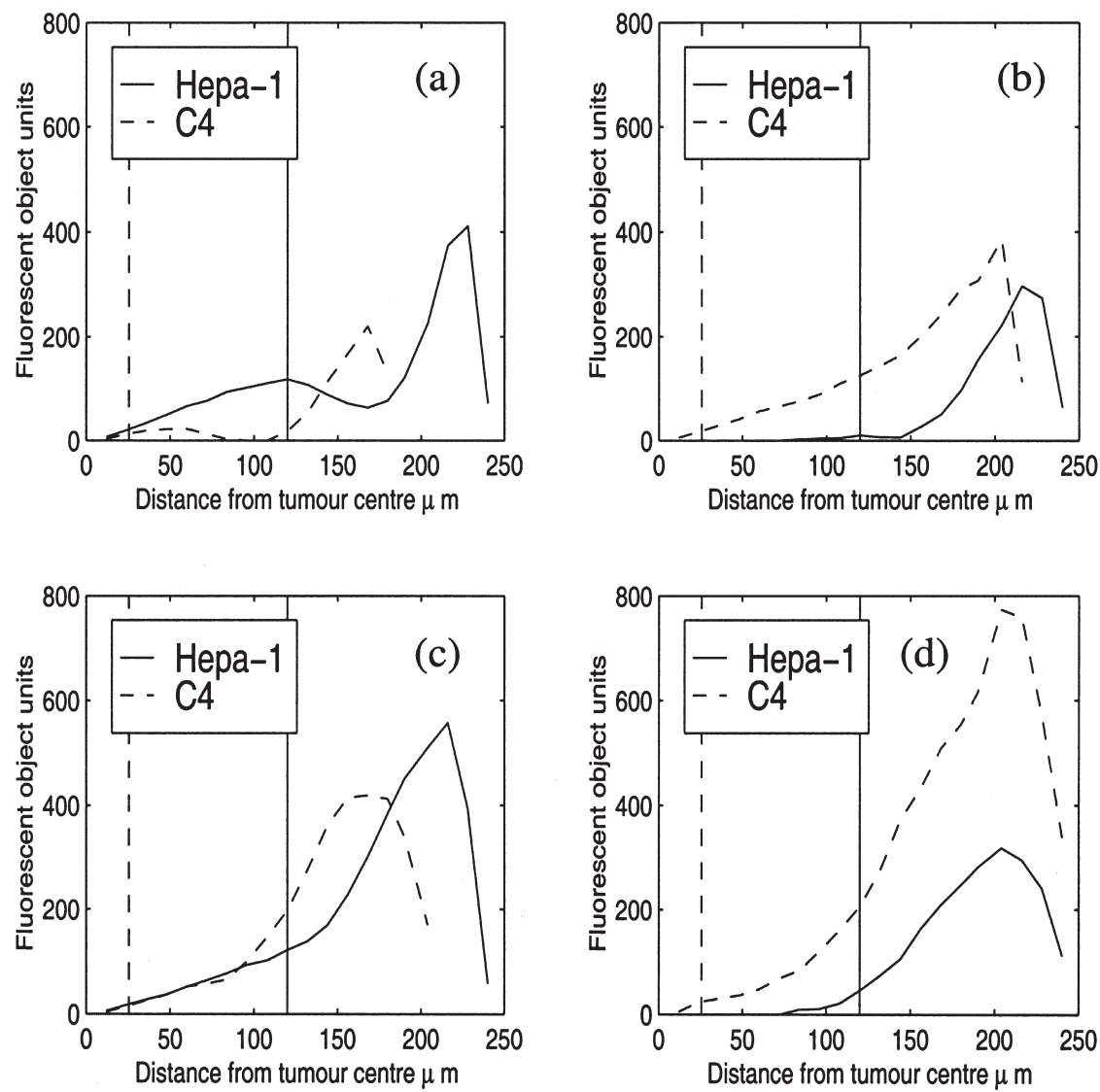

FIGURE 2 Series of figures showing the patterns of macrophage infiltration into 10-day old spheroids cultured from hepa-1 and C4 tumour cells lines after (a) $2 \mathrm{~h}$, (b) $4 \mathrm{~h}$, (c) $6 \mathrm{~h}$ and (d) $12 \mathrm{~h}$ co-culture. Macrophages migrate into the hepa-1 spheroids more rapidly than into $\mathrm{C} 4$ spheroids, their motion being facilitated by chemotaxis. In both cases the peak macrophage density occurs inside the tumour. The solid and dashed vertical lines denote the extent of necrosis for the hepa-1 and C4 spheroids, respectively. Data reproduced from Leek (1999), with permission.

macrophage density occurring a short distance (20$50 \mu \mathrm{m}$ ) from the spheroid surface and thereafter decreasing towards the tumour centre.

The appearance of macrophage infiltrate in the 5-day old spheroids (Fig. 3a) suggests that necrosis is not necessary for macrophage migration. This fact, coupled with the similar patterns of infiltration in the $\mathrm{C} 4$ and hepa-1 spheroids, suggests that for small, non-necrotic spheroids the mechanisms driving macrophage migration are independent of the tumour cells' ability to express hif-1 and related proteins such as VEGF.

Referring to the data for the 10-day old spheroids (Fig. $3 b$ ), which possess regions of hypoxia and necrosis, we note that the macrophages migrate more rapidly into the hepa-1 spheroids. Comparing the profiles for the 5- and 10-day old spheroids, we remark also that the depth of penetration into the older (and larger) spheroids is greater than for the 5-day old spheroids. By contrast, comparing the data for the 10- and 20-day old spheroids (the results for the 20-day old spheroids are presented in Fig. 3c), we note that as the spheroids increase in size, and the extent of central necrosis increases, the degree of macrophage infiltration reduces. This suggests that the size of the hypoxic region plays an important role in macrophage infiltration.
We now summarise those features of Leek's experiments that we expect a realistic mathematical model of macrophage infiltration to exhibit:

- The ability of macrophages to infiltrate all spheroids, regardless of whether they possess a necrotic core;

- The number of macrophages present within the spheroid increases as the number of hypoxicallystressed tumour cells increases;

- Initially macrophages infiltrate more rapidly into spheroids which produce macrophage chemoattractants (hepa-1 spheroids) than those which do not (C4 spheroids);

- The limiting number of macrophages within spheroids which do not produce macrophage chemoattractants is larger than the limiting number in those that do.

As a final remark, the following points should be borne in mind when interpreting the experimental data presented above. Firstly, the sizes and detailed composition of the $\mathrm{C} 4$ and hepa- 1 spheroids are different, the hepa- 1 spheroids being larger and possessing larger necrotic cores (see Table I for details). Second, the spheroids will continue to grow to some extent during the experiments. Finally, the 

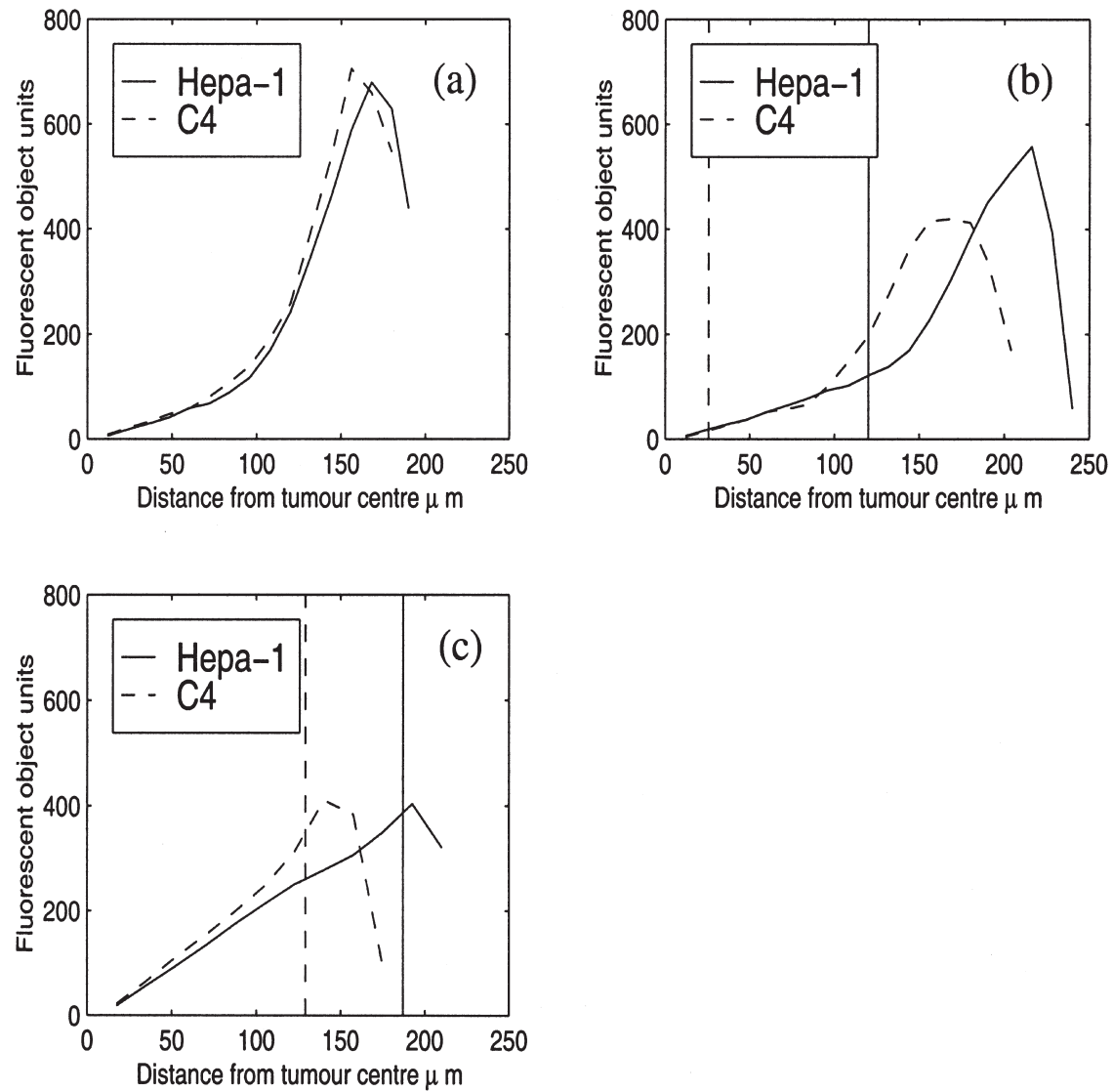

FIGURE 3 Series of figures showing the patterns of macrophage infiltration into (a) 5-day old, (b) 10-day old and (c) 20-day old hepa-1 and C4 spheroids after $6 \mathrm{~h}$ co-culture. As the spheroids age and increase in size, differences in the infiltration patterns into the hepa-1 and C4 spheroids become apparent. The solid and dashed vertical lines denote the extent of necrosis for the hepa-1 and C4 spheroids, respectively. Data reproduced from Leek (1999), with permission.

data is subject to experimental errors, which means that some of the measurements are less reliable than others (see for example the distribution of macrophages within the $\mathrm{C} 4$ spheroids after $12 \mathrm{~h}$ co-culture). Each of these factors makes it difficult to state, with confidence, the mechanisms responsible for the different infiltration patterns seen in Figs. 2 and 3. One advantage of using a mathematical model is that such discrepancies may be eliminated.

\section{THE MATHEMATICAL MODEL}

In this section we develop a mathematical model that describes the migration of macrophages into a sphericallysymmetric, spatially-structured avascular tumour. The model is used to determine whether observed differences in infiltration patterns into spheroids from two tumour cell lines may be due to differences in their rates of expression of a single, generic chemoattractant, with one cell line (hepa-1 spheroids) expressing the chemoattractant under hypoxia and the second (C4 spheroids) not (Leek et al., 1997). The key physical variables employed in the model are the oxygen concentration $c$, the macrophage chemoattractant concentration $\omega$ and the macrophage cell density $m$. All variables are supposed to depend on time $t$, distance from the tumour centre $r$, the radius of the tumour $R$ and its spatial structure. Partial differential equations governing the evolution of $c, \omega$ and $m$, which may be derived by applying mass balances to each species, are presented in turn below. They are solved in conjunction with equations which define the tumour's spatial structure.

TABLE I The average radius and percentage necrosis of the tumour spheroids used to generate the experimental results presented in Figs. 1 and 2 are stated in this table

\begin{tabular}{lcccr}
\hline Spheroid age (days) & Cell type & Tumour radius, $R(\mu \mathrm{m})$ & Necrotic radius, $R_{\mathrm{N}}(\mu \mathrm{m})$ & Percentage necrosis (by volume) \\
\hline 5 & Hepa-1 & 190 & 0 & 0.0 \\
& C4 & 180 & 0 & 0.0 \\
10 & Hepa-1 & 240 & 120 & 12.5 \\
& C4 & 204 & 25.5 & 0.2 \\
20 & Hepa-1 & 210 & 187 & 70.6 \\
& C4 & 157 & 129 & 55.5 \\
\hline
\end{tabular}




\section{The Oxygen Concentration, $\mathbf{c}(\mathrm{r}, \mathrm{t})$}

When applying mass conservation to the oxygen concentration, which is supplied from the fluid surrounding the spheroid, we assume that diffusion drives its motion into the tumour and that it is consumed by live (i.e. non-necrotic) tumour cells as it diffuses towards the spheroid's centre. Combining these effects we obtain the following word equation:

$$
\begin{aligned}
& \text { (Rate of change of oxygen concentration) } \\
& =\text { (motion due to diffusion) } \\
& \quad-\text { (rate of consumption by live tumour cells). }
\end{aligned}
$$

Denoting by $D_{c}$ the assumed constant oxygen diffusion coefficient and by $\lambda_{0}$ the assumed constant rate at which viable (i.e. non-necrotic) tumour cells consume oxygen, we rewrite the word equation in the following form:

$$
\frac{\partial c}{\partial t}=\frac{D_{c}}{r^{2}} \frac{\partial}{\partial r}\left(r^{2} \frac{\partial c}{\partial r}\right)-\lambda_{0} H\left(c-c_{\mathrm{N}}\right) .
$$

In Eq. (1), $H(\cdot)$ denotes the Heaviside step-function $(H(x)=1$ if $x \geq 0, H(x)=0$ if $x<0)$ and $c_{\mathrm{N}}$ denotes the threshold oxygen concentration below which tumour cells are no longer viable ( $c_{\mathrm{N}}$ marks the transition to necrosis). Thus the term $-\lambda_{0} H\left(c-c_{\mathrm{N}}\right)$ limits oxygen consumption to non-necrotic regions where the tumour cells are alive.

\section{The Spheroid's Spatial Structure}

Since the time over which macrophage migration takes place $(\sim 12 \mathrm{~h})$ is much shorter than the tumour doubling time ( $\sim 1$ week) we assume, for simplicity, that during the experiments the spheroid does not grow i.e. its spatial structure is fixed and $R \sim$ constant. Referring to Fig. 3, we note that the tumour's spatial composition changes as its size increases. In order to describe the spheroid's spatial structure, we now introduce $r=R_{\mathrm{H}}$ and $r=R_{\mathrm{N}}$, where $0 \leq R_{\mathrm{N}} \leq R_{\mathrm{H}}<R$, to denote the boundaries between proliferating, hypoxic and necrotic regions. The location of these interfaces is defined implicitly in terms of the threshold oxygen concentrations $c_{\mathrm{H}}, c_{\mathrm{N}}$ in the following way:

$$
\begin{gathered}
R_{\mathrm{H}}=0 \quad \text { if } c>c_{\mathrm{H}} \forall r \in(0, R) ; \\
\text { otherwise } r=R_{\mathrm{H}} \text { when } c=c_{\mathrm{H}}, \\
R_{\mathrm{N}}=0 \quad \text { if } c>c_{\mathrm{N}} \forall r \in(0, R) ; \\
\text { otherwise } r=R_{\mathrm{N}} \text { when } c=c_{\mathrm{N}} .
\end{gathered}
$$

We note that Eqs. (2) and (3) define $R_{\mathrm{H}}$ and $R_{\mathrm{N}}$ uniquely provided that $c$ is a monotonic increasing function of $r$.

\section{The Chemoattractant Concentration, $\omega(\mathrm{r}, \mathrm{t})$}

The evolution of the tumour-cell-derived, macrophage chemoattractant is assumed to be similar in form to that of the oxygen, with diffusion driving its motion, hypoxicallystressed tumour cells acting as sources of $\omega$, and natural decay dominating its removal from the system. Combining these ideas yields the following word equation:

$$
\begin{aligned}
& \text { (Rate of change of chemoattractant concentration) } \\
& =\text { (motion due to diffusion) } \\
& \quad+\text { (rate of production by hypoxic tumour cells) } \\
& \quad-\text { (rate of natural decay). }
\end{aligned}
$$

If we denote by $D_{\omega}, \lambda_{1}$ and $\lambda_{2}$, respectively, the chemoattractant's assumed constant diffusion coefficient, its rate of production by hypoxic tumour cells and its rate of natural decay, then the above word equation can be rewritten as:

$$
\begin{aligned}
\frac{\partial \omega}{\partial t}= & \frac{D_{\omega}}{r^{2}} \frac{\partial}{\partial r}\left(r^{2} \frac{\partial \omega}{\partial r}\right)+\lambda_{1} H\left(c_{\mathrm{H}}-c\right) H\left(c-c_{\mathrm{N}}\right) \\
& -\lambda_{2} \omega .
\end{aligned}
$$

In Eq. (4), $c_{\mathrm{H}}$ is the threshold oxygen concentration which marks the transition from tumour cell proliferation (where $c>c_{\mathrm{H}}$ ) to hypoxia and the cessation of proliferation (where $\mathrm{c}<c_{\mathrm{H}}$ ). The parameter $c_{\mathrm{N}}$ marks the transition oxygen concentration at which cells switch from being hypoxic $\left(c_{\mathrm{N}}<c<c_{\mathrm{H}}\right)$ to being necrotic ( $c \leq$ $\left.c_{\mathrm{N}}\right)$. Thus the factor $\lambda_{1} H\left(c_{\mathrm{H}}-c\right) H\left(c-c_{\mathrm{N}}\right)$ localises chemoattractant production to the region of the tumour containing hypoxically-stressed (i.e. viable, non-proliferating) cells. We remark that $c_{\mathrm{H}} \geq c_{\mathrm{N}}$ i.e. as the oxygen concentration is progressively diminished, hypoxia will be initiated prior to necrosis.

\section{The Macrophage Cell Density, $m(r, t)$}

When considering the evolution of the macrophage cell density, proliferation and death may be neglected since they occur over a longer timescale ( $\sim$ weeks) than the timescale of the experiments ( $\sim$ hours (Leek, 1999)). Under the additional assumption that the dominant factors governing the macrophage population are random motion and chemotaxis (Bottazzi et al., 1983; Bottazzi et al., 1985), we obtain the following word equation for the macrophage cell density:

$$
\begin{aligned}
& \text { (Rate of change of macrophage density) } \\
& =\text { (motion due to random motion) } \\
& \quad+\text { (motion due to chemotaxis). }
\end{aligned}
$$

Denoting by $\mu(c)$ and $\chi(c)$ the random motion and chemotaxis coefficients, the word equation can be 
rewritten as:

$$
\frac{\partial m}{\partial t}=\frac{1}{r^{2}} \frac{\partial}{\partial r}\left(r^{2} \mu(c) \frac{\partial m}{\partial r}\right)-\frac{1}{r^{2}} \frac{\partial}{\partial r}\left(r^{2} \chi(c) m \frac{\partial \omega}{\partial r}\right) .
$$

Experimental results presented by Negus et al. show that macrophage migration is significantly inhibited under hypoxia due to the production of macrophage inhibitory factor (MIF) (Negus et al., 1998). Rather than introducing an additional dependent variable to describe the local concentration of MIF we reproduce the immobilising effect that it exerts on macrophages by making $\mu$ and $\chi$ depend on the oxygen concentration $c$ in an appropriate manner: specifically, both $\mu$ and $\chi$ decrease as the environment becomes more hypoxically-stressed (i.e. the oxygen concentration falls), but remain non-negative for all values of $c$. Thus in Eq. (5) we fix

$$
\begin{gathered}
\mu(c)=\mu\left(\frac{c-c_{\mathrm{N}}}{c_{\infty}+c-2 c_{\mathrm{N}}}\right) H\left(c-c_{\mathrm{N}}\right) \text { and } \\
\chi(c)=\chi\left(\frac{c-c_{\mathrm{N}}}{c_{\infty}+c-2 c_{\mathrm{N}}}\right) H\left(c-c_{\mathrm{N}}\right),
\end{gathered}
$$

where $c_{\infty}$ denotes the oxygen concentration external to the spheroid.

We remark that the inclusion of step-functions in Eqs. (1), (4) and (6) is designed to facilitate the construction of explicit model solutions (see "Mathematical analysis of the model"). When constructing numerical simulations of the model equations (see "The numerical results"), the step-functions are replaced by equivalent, smooth, tanhfunctions.

\section{Boundary and Initial Conditions}

In order to close Eq. (1)-(5), the following boundary and initial conditions are imposed:

$$
\begin{gathered}
\frac{\partial c}{\partial r}=0=\frac{\partial \omega}{\partial r} \quad \text { at } r=0, \\
c=c_{\infty}, \quad D_{\omega} \frac{\partial \omega}{\partial r}=-h_{\omega} \omega, \\
\frac{1}{2}\left(\mu \frac{\partial m}{\partial r}-\chi m \frac{\partial \omega}{\partial r}\right)=h_{m}\left(m_{\infty}-m\right) \quad \text { at } r=R, \\
c, \frac{\partial c}{\partial r}, \omega, \frac{\partial \omega}{\partial r}, m, \frac{\partial m}{\partial r} \quad \text { continuous across } \quad r=R_{\mathrm{H}}, \\
c, \frac{\partial c}{\partial r}, \omega, \frac{\partial \omega}{\partial r} \quad \text { continous across } r=R_{\mathrm{N}}, \\
\mu \frac{\partial m}{\partial r}-\chi m \frac{\partial \omega}{\partial r}=0 \quad \text { at } \quad r=\max \left(0, R_{\mathrm{N}}\right), \\
c(r, 0)=c_{i n}(r), \quad \omega(r, 0)=0, \quad m(r, 0)=m_{i n}(r) .
\end{gathered}
$$

Equations (7) ensure symmetry of $c$ and $\omega$ about the spheroid centre, $r=0$. In Eq. (8), $c_{\infty}$ and $m_{\infty}$ denote, respectively, the assumed constant oxygen concentration and macrophage cell density exterior to the spheroid. The assumed constant parameters $h_{w}$ and $h_{m}$ describe the permeability of the tumour boundary to the chemoattractant and macrophages. The first of Eq. (8) guarantees continuity of $c$ across $r=R$ (this corresponds to assuming that the tumour is highly permeable to oxygen). The other equations define the fluxes of $\omega$ and $m$ across $r=R$. Equations (9) ensure continuity of $c, \omega, m$ and their first partial derivatives across $r=R_{\mathrm{H}}$. Equations (10) ensure continuity of $c, \omega$ and their first partial derivatives across $r=R_{\mathrm{N}}$. Equation (11) states that when $R_{\mathrm{N}}=0$ there is symmetry of $m$ about $r=0$ and that when $R_{\mathrm{N}}>0$ there is no flux of macrophages into the necrotic core. We remark that the boundary conditions (9)-(11) are needed to construct analytical solutions when step-functions are used in Eqs. (1), (4) and (6) (the numerical solutions presented in "The numerical results" are obtained by using smooth (infinitely differentiable) tanh-functions to approximate the step-functions: in this situation, boundary conditions (9)-(11) and Eqs. (2) and (3) are obsolete). Finally, Eqs. (12) define the initial distributions of $c, \omega, m$ within the spheroid.

\section{Nondimensionalisation}

Before constructing numerical and analytical solutions of our model, it is convenient to recast Eqs. (1)-(12) in terms of dimensionless variables. Thus we introduce

$$
\begin{aligned}
& c^{*}=\frac{c}{c_{\infty}}, \quad \omega^{*}=\frac{\omega}{\Omega}, \quad m^{*}=\frac{m}{M}, \quad r^{*}=\frac{r}{R_{0}} \\
& \text { and } t^{*}=\frac{t}{T}
\end{aligned}
$$

where $\Omega$ is a typical chemoattractant concentration, $M$ is a typical macrophage cell density, $R_{0}$ is a reference lengthscale, and $T$ is a reference time-scale. If we fix

$$
R_{0}^{2}=\frac{D_{c} c_{\infty}}{\lambda_{0}}, \quad T=\frac{R_{0}^{2}}{\mu} \quad \text { and } \quad \Omega=\frac{\mu}{\chi},
$$

then the non-dimensional versions of Eqs. (1)-(5) are

$$
\begin{gathered}
\left(\frac{\mu}{D_{c}}\right) \frac{\partial c^{*}}{\partial t^{*}}=\frac{1}{r^{*}} \frac{\partial}{\partial r^{*}}\left(r^{*} \frac{\partial c^{*}}{\partial r^{*}}\right)-H\left(c^{*}-c_{\mathrm{N}}^{*}\right), \\
R_{H}^{*}=0 \quad \text { if } c^{*}>c_{H}^{*} \forall r^{*} \in\left(0, R^{*}\right) ; \\
\text { otherwise } r^{*}=R_{H}^{*} \text { when } c^{*}=c_{H}^{*}, \\
R_{\mathrm{N}}^{*}=0 \text { if } c^{*}>c_{\mathrm{N}}^{*} \forall r^{*} \in\left(0, R^{*}\right) ; \\
\text { otherwise } r^{*}=R_{\mathrm{N}}^{*} \text { when } c^{*}=c_{\mathrm{N}}^{*},
\end{gathered}
$$




$$
\begin{aligned}
\left(\frac{\mu}{D_{\omega}}\right) \frac{\partial \omega^{*}}{\partial t^{*}}= & \frac{1}{r^{*} 2} \frac{\partial}{\partial r^{*}}\left(r^{*} \frac{\partial \omega^{*}}{\partial r^{*}}\right)+\lambda_{1}^{*} H\left(c_{H}^{*}\right. \\
\frac{\partial m^{*}}{\partial t^{*}}= & \frac{1}{r^{* 2}} \frac{\partial}{\partial r^{*}}\left(\frac{r^{*}\left(c^{*}-c_{\mathrm{N}}^{*}\right)}{1+c^{*}-2 c_{\mathrm{N}}^{*}} H\left(c^{*}-c_{\mathrm{N}}^{*}\right) \frac{\partial m^{*}}{\partial r^{*}}\right) \\
& -\frac{1}{r^{* 2}} \frac{\partial}{\partial r^{*}} \frac{r^{* 2}\left(c^{*}-c_{\mathrm{N}}^{*}\right) m^{*}}{1+c^{*}-2 c_{\mathrm{N}}^{*}} H\left(c^{*}-c_{\mathrm{N}}^{*}\right) \\
& \times \frac{\partial \omega^{*}}{\partial r^{*}}
\end{aligned}
$$

where

$$
\begin{gathered}
R^{*}=\frac{R}{R_{0}}, \quad R_{H}^{*}=\frac{R_{\mathrm{H}}}{R_{0}}, \quad R_{\mathrm{N}}^{*}=\frac{R_{\mathrm{N}}}{R_{0}}, \quad c_{H}^{*}=\frac{c_{\mathrm{H}}}{c_{\infty}}, \\
c_{\mathrm{N}}^{*}=\frac{c_{\mathrm{N}}}{c_{\infty}}, \quad \lambda_{1}^{*}=\frac{\lambda_{1} R_{0}^{2}}{D_{\omega} \Omega}, \quad \lambda_{2}^{*}=\frac{\lambda_{2} R_{0}^{2}}{D_{\omega}} .
\end{gathered}
$$

Since oxygen molecules are typically much smaller than protein molecules (e.g. chemoattractants such as VEGF and MCP-1) which are smaller than individual cells (e.g. macrophages), we anticipate that oxygen will diffuse much more rapidly than the chemoattractant which will, in turn, diffuse more quickly than the macrophages. We combine this physical insight with experimental measurements, which indicate that $D_{c} \sim 10^{-10} \mathrm{~m}^{2} \mathrm{~s}^{-1}$, to estimate that

$$
\frac{\mu}{D_{c}} \sim 10^{-4} \text { and } \frac{\mu}{D_{\omega}} \sim 10^{-2} .
$$

Neglecting terms involving $\mu / D_{\mathrm{c}}$, and $\mu / D_{\omega}$, we recover the usual, quasi-steady, reaction-diffusion equations for the oxygen and chemoattractant concentrations (Adam, 1987; Greenspan, 1972).

Introducing

$$
h_{\omega}^{*}=\frac{h_{\omega} R_{0}}{D_{\omega}}, \quad h_{m}^{*}=\frac{h_{m} R_{0}}{\mu}, \quad m_{\infty}^{*}=\frac{m_{\infty}}{M},
$$

and, henceforth, omitting *s for clarity, we deduce that our nondimensional model equations are given by

$$
\begin{gathered}
0=\frac{1}{r^{2}} \frac{\partial}{\partial r}\left(r^{2} \frac{\partial c}{\partial r}\right)-H\left(c-c_{\mathrm{N}}\right), \\
R_{\mathrm{H}}=0 \quad \text { if } c>c_{\mathrm{H}} \forall r \in(0, R) ; \\
\text { otherwise } r=R_{\mathrm{H}} \quad \text { when } c=c_{\mathrm{H}}, \\
R_{\mathrm{N}}=0 \quad \text { if } c>c_{\mathrm{N}} \forall r \in(0, R) ; \\
\text { otherwise } r=R_{\mathrm{N}} \quad \text { when } c=c_{\mathrm{N}},
\end{gathered}
$$

$$
\begin{aligned}
0=\frac{1}{r^{2}} & \frac{\partial}{\partial r}\left(r^{2} \frac{\partial \omega}{\partial r}\right)+\lambda_{1} H\left(c_{\mathrm{H}}-c\right) H\left(c-c_{\mathrm{N}}\right) \\
& -\lambda_{2} \omega, \\
\frac{\partial m}{\partial t}= & \frac{1}{r^{2}} \frac{\partial}{\partial r}\left(\frac{r^{2}\left(c-c_{\mathrm{N}}\right)}{1+c-2 c_{\mathrm{N}}} H\left(c-c_{\mathrm{N}}\right) \frac{\partial m}{\partial r}\right) \\
& -\frac{1}{r^{2}} \frac{\partial}{\partial r}\left(\frac{r^{2}\left(c-c_{\mathrm{N}}\right) m}{1+c-2 c_{\mathrm{N}}} H\left(c-c_{\mathrm{N}}\right) \frac{\partial \omega}{\partial r}\right) .
\end{aligned}
$$

In a similar fashion, it is possible to show that the boundary and initial conditions transform to give

$$
\frac{\partial c}{\partial r}=0=\frac{\partial \omega}{\partial r} \quad \text { at } r=0,
$$

$$
\begin{gathered}
c=1, \quad \frac{\partial \omega}{\partial r}=-h_{\omega} \omega, \\
\frac{\partial m}{\partial r}-m \frac{\partial \omega}{\partial r}=2 h_{m}\left(m_{\infty}-m\right) \quad \text { at } r=R \\
c, \frac{\partial c}{\partial r}, \omega, \frac{\partial \omega}{\partial r}, m, \frac{\partial m}{\partial r} \quad \text { continuous across } r=R_{\mathrm{H}}, \\
c, \frac{\partial c}{\partial r}, \omega, \frac{\partial \omega}{\partial r} \quad \text { continuous across } r=R_{\mathrm{N}}, \\
\frac{\partial m}{\partial r}-m \frac{\partial \omega}{\partial r}=0 \quad \text { at } r=\max \left(0, R_{\mathrm{N}}\right), \\
m(r, 0)=m_{i n}(r) .
\end{gathered}
$$

We note that since $c$ and $\omega$ satisfy time-independent reaction-diffusion equations, they evolve instantaneously to maintain a quasi-steady equilibrium. As a result, initial conditions for $c$ and $\omega$ may, henceforth, be neglected.

In summary, our non-dimensional model of macrophage infiltration is defined by Eqs. (18)-(28). In the following sections numerical and analytical model solutions are presented which provide insight into the experimental results of "The experimental results". When using the model to explain the observed differences in macrophage infiltration into $\mathrm{C} 4$ and hepa- 1 spheroids, model parameters on which attention will focus are the rate of production of chemoattractant $\lambda_{1}$ and the tumour radius $R$ (see Figs. 2 and 3). In particular, cases for which the tumour cells produce macrophage chemoattractant $\left(\lambda_{1}>0\right)$ correspond to hepa-1 spheroids whereas those for which no chemoattractant is produced correspond to $\mathrm{C} 4$ spheroids.

\section{THE NUMERICAL RESULTS}

The model Eqs. (18)-(28) were solved numerically using a package from the NAG library (routine DO3PCF). This method uses finite difference approximations to perform a 

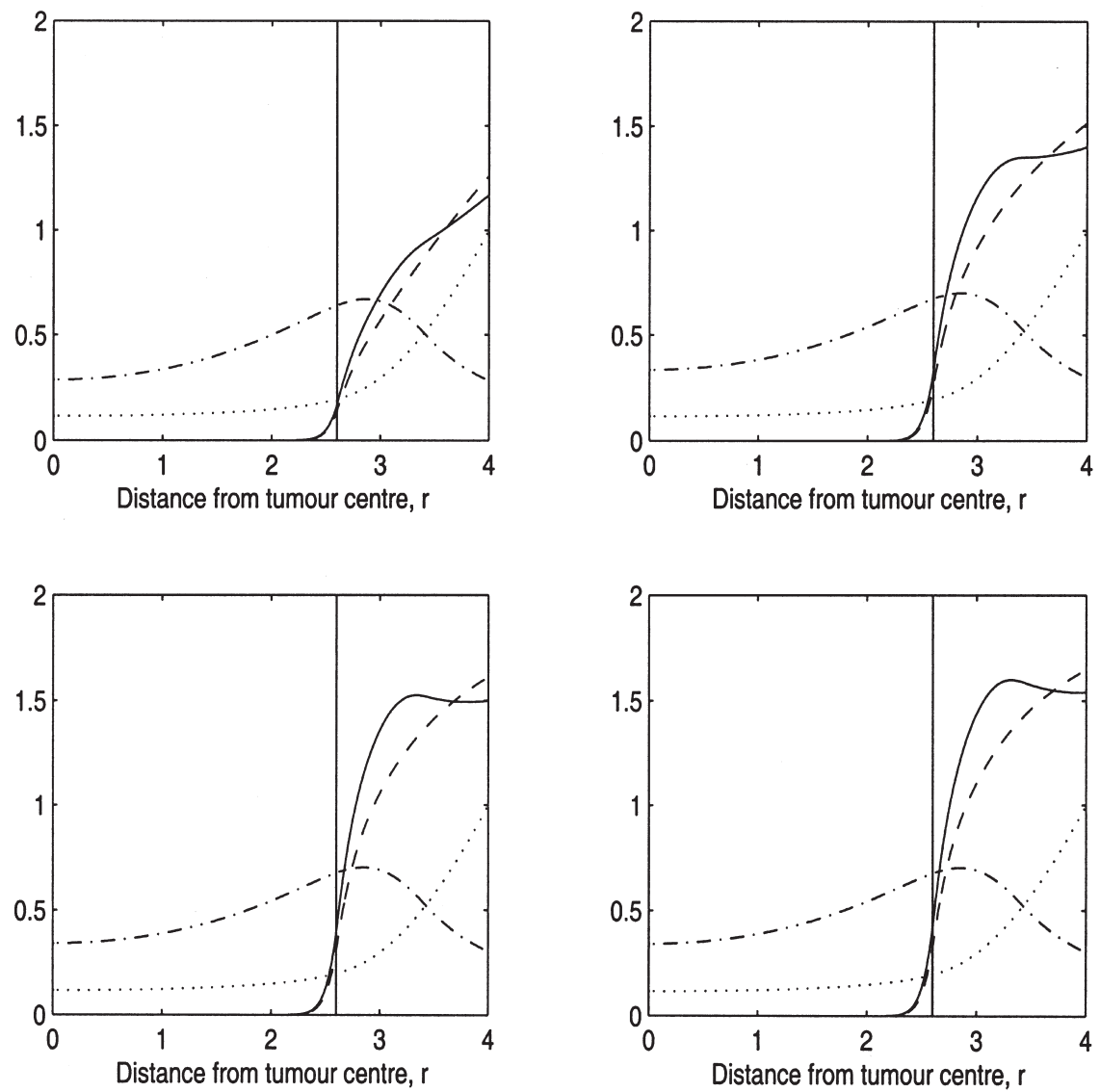

FIGURE 4 Here we present the results of numerical simulations which show how macrophages infiltrate into a well-developed spheroid for two choices of the chemoattractant production rate, $\lambda_{1}=2.0$ (hepa-1 spheroids) and $\lambda_{1}=0.0$ (C4 spheroids). The centre of the tumour is located at $r=0$, the boundary at $r=R$ and macrophage infiltration proceeds from right to left. The dependent variables are plotted at dimensionless times $t=2,4,6,8$. For both the hepa-1 and $\mathrm{C} 4$ spheroids the numbers of infiltrating macrophages increases initially, before settling to equilibrium values, with more macrophages infiltrating the hepa-1 spheroid than the $\mathrm{C} 4$ spheroid. In both cases the macrophages infiltrate as far as the necrotic boundary $\left(R_{\mathrm{N}} \sim 2.6\right)$. For the hepa-1 spheroids the maximum macrophage density occurs inside the tumour whereas for the $\mathrm{C} 4$ spheroids it occurs on the tumour boundary. Key: oxygen concentration (dotted line); chemoattractant concentration (dot-dashed line); macrophage density within hepa-1 spheroids (solid line, $\lambda_{1}=2.0$ ); macrophage density within C4 spheroids (dashed line, $\lambda_{1}=0.0$ ) (the vertical solid line marks the necrotic boundary). Parameter values: $R=4, \lambda_{2}=1.0, h_{\omega}=1.0, h_{m}=0.5, m_{\infty}=2.0, c_{\mathrm{H}}=0.5, c_{\mathrm{N}}=0.2$.

spatial discretisation of the model equations, thereby reducing them to a system of time-dependent ordinary differential equations which are readily integrated (this is the method of lines). In the absence of experimental estimates, parameter values were chosen that produced simulations which were qualitatively similar to the experimental results.

The results presented in Fig. 4 show how the key physical variables evolve during a typical simulation, and relate directly to the experimental results of Fig. 2. The figures show that the oxygen and chemoattractant concentrations rapidly adopt equilibrium profiles, with the oxygen concentration decreasing monotonically towards the tumour centre and defining its spatial structure (see Eqs. (19) and (20)). The maximum chemoattractant concentration occurs within the hypoxic region $\left(R_{\mathrm{N}} \sim\right.$ $2.6<r<R_{\mathrm{H}} \sim 3.4$ ) in which its production is localised. As time evolves, differences in the patterns of macrophage infiltration between the hepa- 1 and $\mathrm{C} 4$ spheroids become apparent. For both cell lines, the macrophages penetrate as far as the necrotic boundary (since macrophages cannot survive within the fluid-filled necrotic core, only a small number ever penetrate into the necrotic core). Also, the total number of infiltrating macrophages increases initially, before settling to equilibrium values, with more macrophages infiltrating the chemoattractant-producing, hepa-1 spheroid than its chemoattractant-deficient, C4 analogue. For early times $(t=2,4)$, both macrophage profiles decrease monotonically with decreasing $r$. For later times $(t=6,8)$, the distribution of macrophages within the $\mathrm{C} 4$ spheroid remains monotonic, whereas the distribution within the hepa-1 spheroid develops an interior maximum inside the hypoxic region. We note that the maximum macrophage density does not coincide with the peak chemoattractant concentration.

As stated above, the numerical simulations are in reasonable qualitative agreement with Leek's experimental results (Leek, 1999). By comparing Figs. 2 and 4 we conclude that the different patterns of macrophage infiltration into hepa- 1 and $\mathrm{C} 4$ tumour spheroids may be due to differences in their rates of production of macrophage chemoattractant. Given that the numerical 

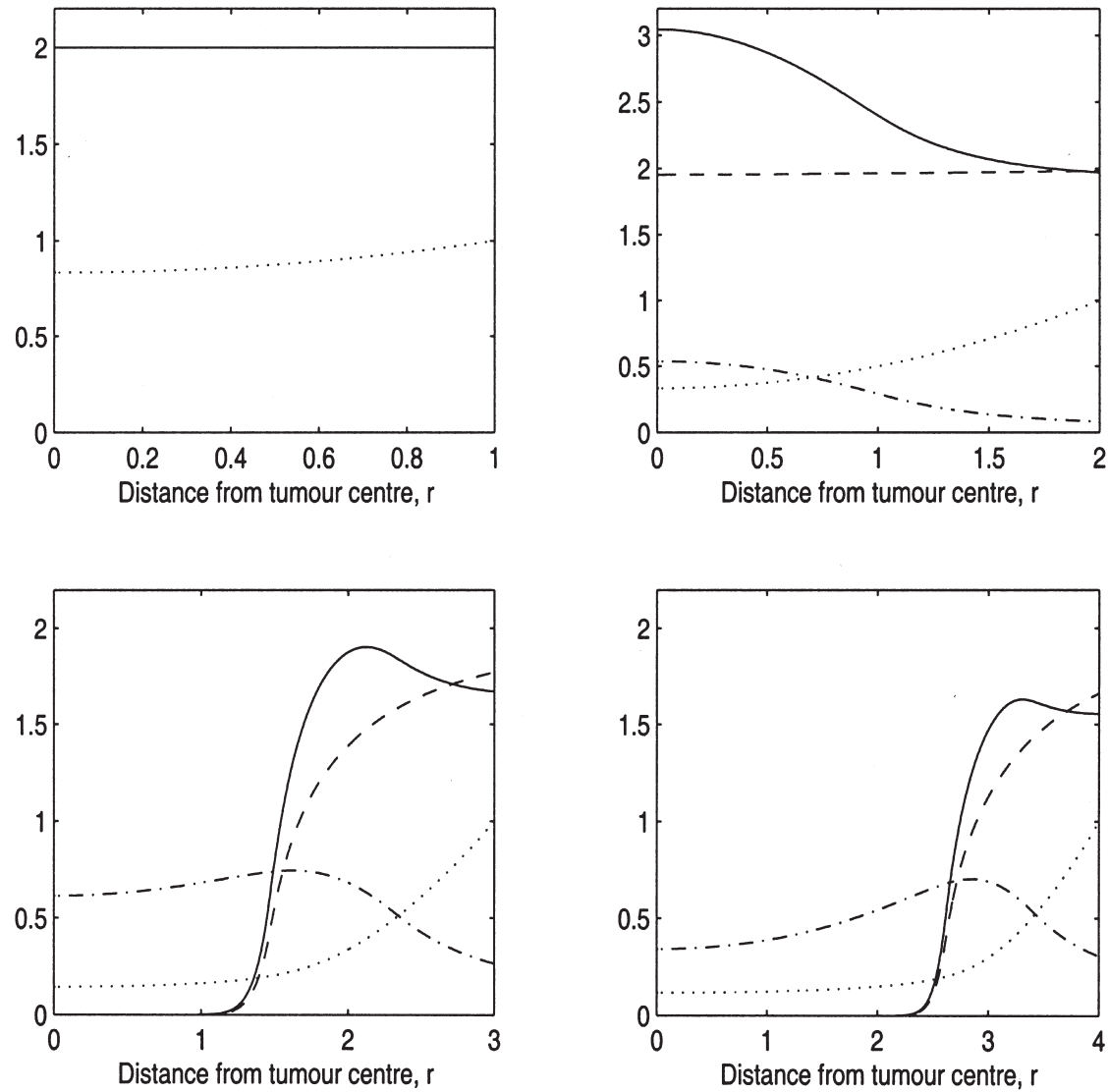

FIGURE 5 Here we present the numerical results showing how size affects macrophage infiltration into hepa- 1 spheroids $\left(\lambda_{1}=2.0\right)$ and C4 spheroids $\left(\lambda_{1}=0.0\right)$ at time $t=10$ (in dimensionless units). For small spheroids $(R=1)$ the infiltration patterns are identical: in both cases the macrophages migrate by random motion alone because there is no hypoxia and, hence, no macrophage chemoattractant present. Once a hypoxic region develops, different patterns of infiltration are observed: for intermediate-sized spheroids $(R=2)$, which possess a hypoxic region and no necrosis, the macrophages are distributed uniformly within the $\mathrm{C} 4$ spheroids whereas the peak macrophage density within the hepa- 1 spheroids coincides with the maximum chemoattractant concentration which occurs at $r=0$. For well-developed spheroids $(R=3,4)$, which possess hypoxic and necrotic regions, the macrophages halt at the necrotic boundary in all cases. For the hepa-1 spheroids the maximum macrophage density occurs inside the tumour whereas for the $\mathrm{C} 4$ spheroids it occurs on the tumour boundary. Key: oxygen concentration (dotted line); chemoattractant concentration (dot-dashed line); macrophage density within hepa-1 spheroids (solid line, $\lambda_{1}=2.0$ ); macrophage density within C4 spheroids (dashed line, $\lambda_{1}=0.0$ ). Parameter values, other than $R$ and $t$ : as per Fig. 4.

simulations predict that, in the absence of chemoattractant, the maximum macrophage density occurs on the tumour boundary and that the experimentally-determined, maximum macrophage density for the $\mathrm{C} 4$ spheroids occurs inside the tumour (subject to experimental error), we predict that cells within the $\mathrm{C} 4$ spheroids are producing macrophage chemoattractants, albeit at lower levels than the hepa-1 spheroids.

The results presented in Fig. 5 show how tumour size and, therefore, spatial structure affect macrophage infiltration into chemoattractant-producing, hepa-1 spheroids $\left(\lambda_{1}, \omega>0\right)$ and chemoattractant-deficient, C4 spheroids $\left(\lambda_{1}=\omega=0\right)$, and are included for comparison with the experimental results of Fig. 2. Figure 5 should also be viewed in parallel with Fig. 6, a bifurcation diagram showing how the tumour's spatial structure, as defined by the position of the hypoxic and necrotic boundaries $R_{\mathrm{H}}$ and $R_{\mathrm{N}}$, changes with the tumour radius $R$. In each graph the oxygen and chemoattractant concentrations are plotted together with the corresponding macrophage density profiles for the hepa- 1 and $\mathrm{C} 4$ spheroids at time $t=10$. For small, well-oxygenated spheroids $\left(R=1.0, R_{\mathrm{H}}=0.0=R_{\mathrm{N}}\right)$, the hepa- 1 and $\mathrm{C} 4$ infiltration patterns are identical because, in the absence of hypoxia, the hepa-1 spheroids do not produce chemoattractant and, as a result, in both cases the macrophages migrate by random motion. By $t=10$ the macrophages are uniformly distributed throughout the tumour volume. For larger tumours, which possess a hypoxic region, different patterns of macrophage infiltration become apparent. For example, macrophages infiltrating a $\mathrm{C} 4$ spheroid which possesses a central region of hypoxia $(R=$ $2.0, R_{\mathrm{H}}=1.0, R_{\mathrm{N}}=0.0$ ) are uniformly distributed by $t=$ 10. By contrast, macrophages infiltrating a hepa-1 spheroid of the same size accumulate at its centre, where the maximum chemoattractant concentration occurs. For well-developed $\mathrm{C} 4$ and hepa-1 spheroids which possess hypoxic and necrotic regions $(R=3.0$, $\left.R_{\mathrm{H}}=2.3, R_{\mathrm{N}}=1.5 ; R=4.0, R_{\mathrm{H}}=3.4, R_{\mathrm{N}}=2.7\right)$, the macrophages always halt at the necrotic boundary. The 


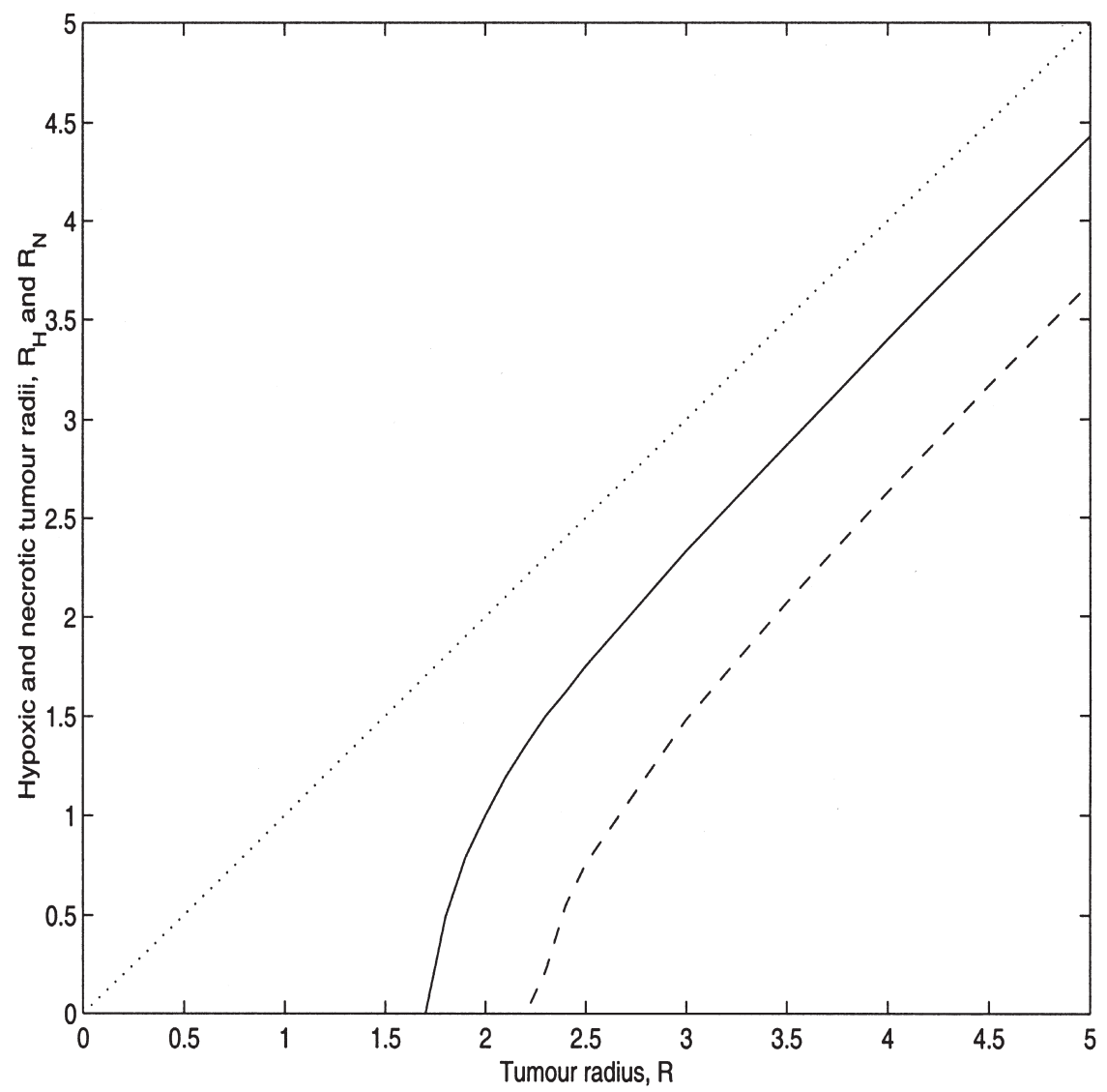

FIGURE 6 Here we show how the tumour's spatial structure changes as its radius $R$ increases. The size of the hypoxic and necrotic radii $R_{\mathrm{H}}$ and $R_{\mathrm{N}}$ are plotted against $R$. By also including the $45^{\circ}$ line $R=R$, we note that as $R$ increases the widths of the proliferating and hypoxic regions tend to constant values, implying that the proportion of the tumour occupied by proliferating and hypoxic cells decreases with increasing $R$. Key: hypoxic radius, $R_{\mathrm{H}}$ (solid line); necrotic radius, $R_{\mathrm{N}}$ (dashed line); outer tumour radius, $R$ (dotted line). Parameter values: as per Fig. 4.

limiting profiles are qualitatively similar to those presented in Fig. 4 and described above. In particular, the macrophage density within a $\mathrm{C} 4$ spheroid decreases progressively from its boundary towards its centre whereas within a hepa-1 spheroid the macrophages accumulate within the hypoxic region.

Referring to Figs. 4 and 5, it is clear that the mathematical model predicts that, at all times, the chemoattractant-producing, hepa-1 spheroids recruit larger numbers of macrophages than chemoattractantdeficient, C4 spheroids of the same size and spatial structure. It is less obvious how, once the system has settled to a steady state (by $t \sim 8-10$ dimensionless units), the total number of macrophages within the spheroid depends on its radius $R$. To clarify this matter, in Fig. 7 we sketch

$$
M\left(t=10 ; R, \lambda_{1}\right)=\int_{R_{\mathrm{N}}}^{R} m(r, t=10) r^{2} \mathrm{~d} r
$$

for different choices of $R$ and $\lambda_{1}$, the total number of macrophages within hepa-1 $\left(\lambda_{1}=2.0\right)$ and $\mathrm{C} 4$ spheroids $\left(\lambda_{1}=0.0\right)$ being plotted for each value of $R$. We note that $M\left(t=10 ; R, \lambda_{1}\right)$ increases with increasing $R$ and $\lambda_{1}$.
The increase in the total number of infiltrating macrophages that is observed when the tumour radius $R$ increases may be due simply to the increase in volume within which the macrophages are distributed. Thus in Fig. 8 we show how the mean macrophage density

$$
\rho_{m}=\frac{M\left(t=10 ; R, \lambda_{1}\right)}{R^{3}-R_{\mathrm{N}}^{3}}
$$

within hepa-1 and $\mathrm{C} 4$ spheroids varies with $R$. For small spheroids, which possess neither hypoxic nor necrotic regions, the macrophages are distributed uniformly throughout the tumour volume (see Fig. 5, with $R=1$ ) and the mean macrophage densities within the hepa-1 and C4 spheroids are identical. As $R$ increases this situation persists until, when a hypoxic region forms, the mean densities in the two types of tumours diverge. Under hypoxia, the hepa- 1 tumour cells produce chemoattractant and the corresponding value of $\rho_{m}$ increases with $R$ until necrosis is initiated ( $R \sim 2.3$, see Fig. 6). By contrast, for the $\mathrm{C} 4$ spheroids $\rho_{m}$ remains approximately constant as $R$ increases until necrosis occurs. Thereafter, the mean macrophage density within both the hepa-1 and $\mathrm{C} 4$ spheroids decreases with increasing $R$, with the mean 


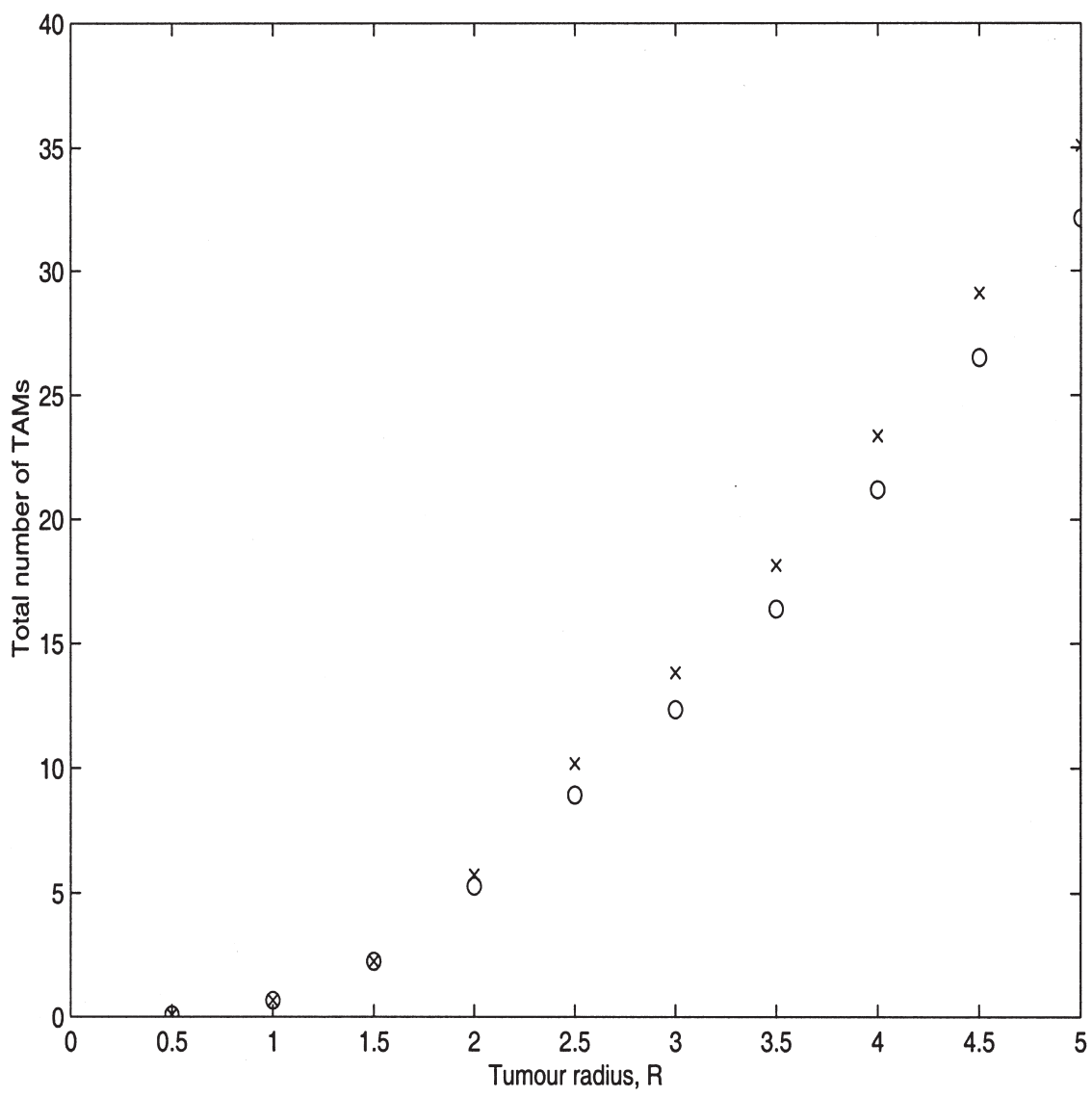

FIGURE 7 Here we show that the total number of TAMs $\left(M\left(t=10 ; R, \lambda_{1}\right)\right)$ that have infiltrated hepa-1 $\left(\lambda_{1}=2.0\right)$ and C4 spheroids $\left(\lambda_{1}=0.0\right)$ of radius $R$ at time $t=10$ (dimensionless units) increases with $R$. We note that the hepa-1 spheroids attract more macrophages than their $\mathrm{C} 4$ counterparts. Key: hepa-I spheroids $\left(\times, \lambda_{1}=2.0\right), \mathrm{C} 4$ spheroids $\left(O, \lambda_{1}=0.0\right)$. Parameter values: as per Fig. 4.

density in the hepa-1 spheroids remaining larger than the mean density in the equivalent $\mathrm{C} 4$ spheroid.

Leek's experiments were carried out in still culture, i.e. the oxygen-rich solution into which the macrophages and spheroids were initially introduced was held stationary. As a result, less than one hour after the start of a given experiment, the spheroids and macrophages had settled, with the macrophages forming a layer that coats the bottom of the well and acts as a rich source of macrophages which continuously penetrate the spheroid through the point of contact. When similar experiments were carried out in an agitated, i.e. well-mixed, environment, similar patterns of macrophage infiltration were observed (results not shown) but the total number of infiltrating macrophages was markedly reduced. This is because when the solution is agitated or stirred the macrophages are diluted throughout the solution, and, in consequence, the rate at which macrophages make contact with spheroids is reduced. Using our mathematical model it is possible to switch between the agitated and still conditions by changing $m_{\infty}$, the density of macrophages outside the spheroid, in Eq. (24). In Fig. 9 we show that $M\left(t=10 ; R, \lambda_{1}\right)$ (i.e. the total number of macrophages that have infiltrated well-developed, hepa-1 and C4 spheroids (of radius $R=4$ ) at time $t=10$ ) varies with $m_{\infty}$. As anticipated, $M\left(t=10 ; R, \lambda_{1}\right)$ increases with $m_{\infty}$ with the hepa-1 spheroids consistently attracting more macrophages than the $\mathrm{C} 4$ spheroids. When the total number of infiltrating macrophages is rescaled with $m_{\infty}$ and the resulting normalised integral

$$
M_{\text {norm }}=\frac{M\left(t=10 ; R, \lambda_{1}\right)}{m_{\infty}}
$$

plotted against $m_{\infty}$ we see that $M_{\text {norm }}$ is approximately constant and independent of $m_{\infty}$ for both types of spheroids. Thus we conclude that the total number of macrophages infiltrating a spheroid depends approximately linearly on $m_{\infty}$.

\section{MATHEMATICAL ANALYSIS OF THE MODEL}

Referring to the model equations, we note that $c, R_{\mathrm{H}}$ and $R_{\mathrm{N}}$ are defined independently of $\omega$ and $m$. Moreover, for fixed values of $c_{\mathrm{H}}$ and $c_{\mathrm{N}}$, the threshold oxygen concentrations marking the transition from tumour cell proliferation $\left(c>c_{\mathrm{H}}\right)$ to hypoxic $\left(c<c_{\mathrm{H}}\right)$ and from viable cells $\left(c>c_{\mathrm{N}}\right)$ to necrotic cells $\left(c<c_{\mathrm{N}}\right)$, it is possible to solve Eqs. (18)-(20) subject to Eqs. (23) and (24) and to obtain expressions for $c, R_{\mathrm{H}}$ and $R_{\mathrm{N}}$ in terms of the spheroid radius $R$. In case $\mathrm{I}$, the radius of the spheroid is small enough that its nutritional demands are met by diffusion and all cells are proliferating. In case II, the 


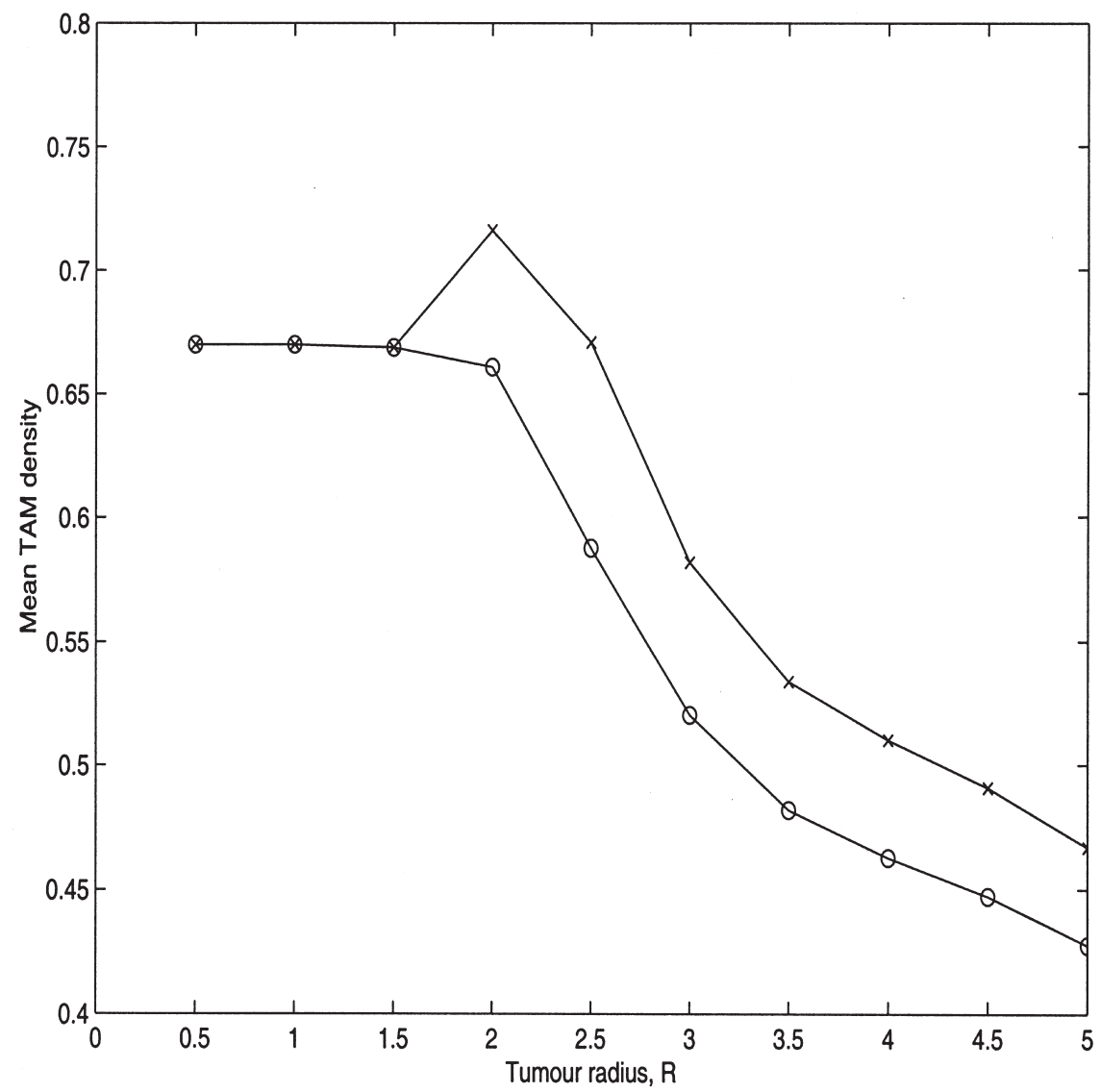

FIGURE 8 Here we show how spheroid size affects the mean macrophage density $\left(\rho_{\mathrm{m}}\right)$ within hepa-1 $\left(\lambda_{1}=2.0\right)$ and $\mathrm{C} 4$ spheroids $\left(\lambda_{1}=0.0\right)$ at time $t=10$ (dimensionless units). For small spheroids, which do not possess hypoxic or necrotic regions, the mean macrophage density within the hepa-1 and $\mathrm{C} 4$ spheroids are identical. Once a hypoxic region develops $(R \sim 1.8)$, the hepa-1 spheroids start to produce macrophage chemoattractant and the mean macrophage densities in the two types of spheroids differ, being larger within the hepa-1 spheroids than within the C4 spheroids. For the hepa-1 spheroids the increase in the mean macrophage density coincides with the appearance of the hypoxic region. Once a necrotic core forms $(R \sim 2.3)$, the mean macrophage density starts to fall. For the $\mathrm{C} 4$ spheroids, the mean macrophage density remains approximately constant until the necrotic core forms. Thereafter, as for the hepa- 1 spheroids, the mean macrophage density decreases with increasing $R$. Key: hepa- 1 spheroids $(\times)$, C4 spheroids $(O)$. Parameter values: as per Fig. 4.

spheroid is larger, and so the amount of nutrient reaching its centre is small enough to halt cell division there. The resulting spheroid comprises a central hypoxic core and a proliferating rim. In case III, we consider a well-developed spheroid for which cells near the centre are so nutrient deficient that they are no longer viable and a necrotic core forms. Cells near the periphery have adequate nutrient and are proliferating. The annulus between the proliferating rim and the necrotic core contains hypoxic cells.

Case I. Tumour comprises proliferating region only $\left(0<R^{2}<6\left(1-c_{\mathrm{H}}\right)\right)$.

$$
c(r)=1-\left(R^{2}-r^{2}\right) / 6 \quad \text { and } \quad R_{\mathrm{H}}=R_{\mathrm{N}}=0 .
$$

Case II. Tumour comprises proliferating and hypoxic regions $\left(6\left(1-c_{\mathrm{H}}\right)<R^{2}<6\left(1-c_{\mathrm{N}}\right)\right)$.

$$
\begin{gathered}
c(r)=1-\left(R^{2}-r^{2}\right) / 6, \quad R_{\mathrm{N}}=0 \quad \text { and } \\
R_{H}^{2}=R^{2}-6\left(1-c_{\mathrm{H}}\right) .
\end{gathered}
$$

Case III. Tumour comprises proliferating, hypoxic and necrotic regions $\left(6\left(1-c_{\mathrm{N}}\right)<R^{2}\right)$.

$$
\begin{gathered}
c(r)=\left\{\begin{array}{cl}
c_{\mathrm{N}} & \text { for } 0<r<R_{\mathrm{N}} \\
c_{\mathrm{N}}-R_{N}^{2} / 2+r^{2} / 6+R_{N}^{3} / 3 r & \text { for } R_{\mathrm{N}}<r<R
\end{array}\right. \\
1-c_{\mathrm{N}}=\frac{R^{2}}{6}-\frac{R_{N}^{2}}{2}+\frac{R_{N}^{3}}{3 R} \text { and } \\
c_{\mathrm{H}}-c_{\mathrm{N}}=\frac{R_{H}^{2}}{6}-\frac{R_{N}^{2}}{2}+\frac{R_{N}^{3}}{3 R_{\mathrm{H}}} .
\end{gathered}
$$

The above expressions for $R_{\mathrm{H}}$ and $R_{\mathrm{N}}$ may be used to construct a bifurcation diagram showing how the tumour's spatial structure changes with $R$. The resulting figure is almost identical to the numerically-constructed diagram presented in Fig. 6 and is, therefore, not included here. We now use these results to determine how the long-time distributions of the chemoattractant and macrophages depend on the tumour's size and spatial structure. For notational simplicity, we assume, henceforth, that $\lambda_{2}=1$ in Eq. (21). 

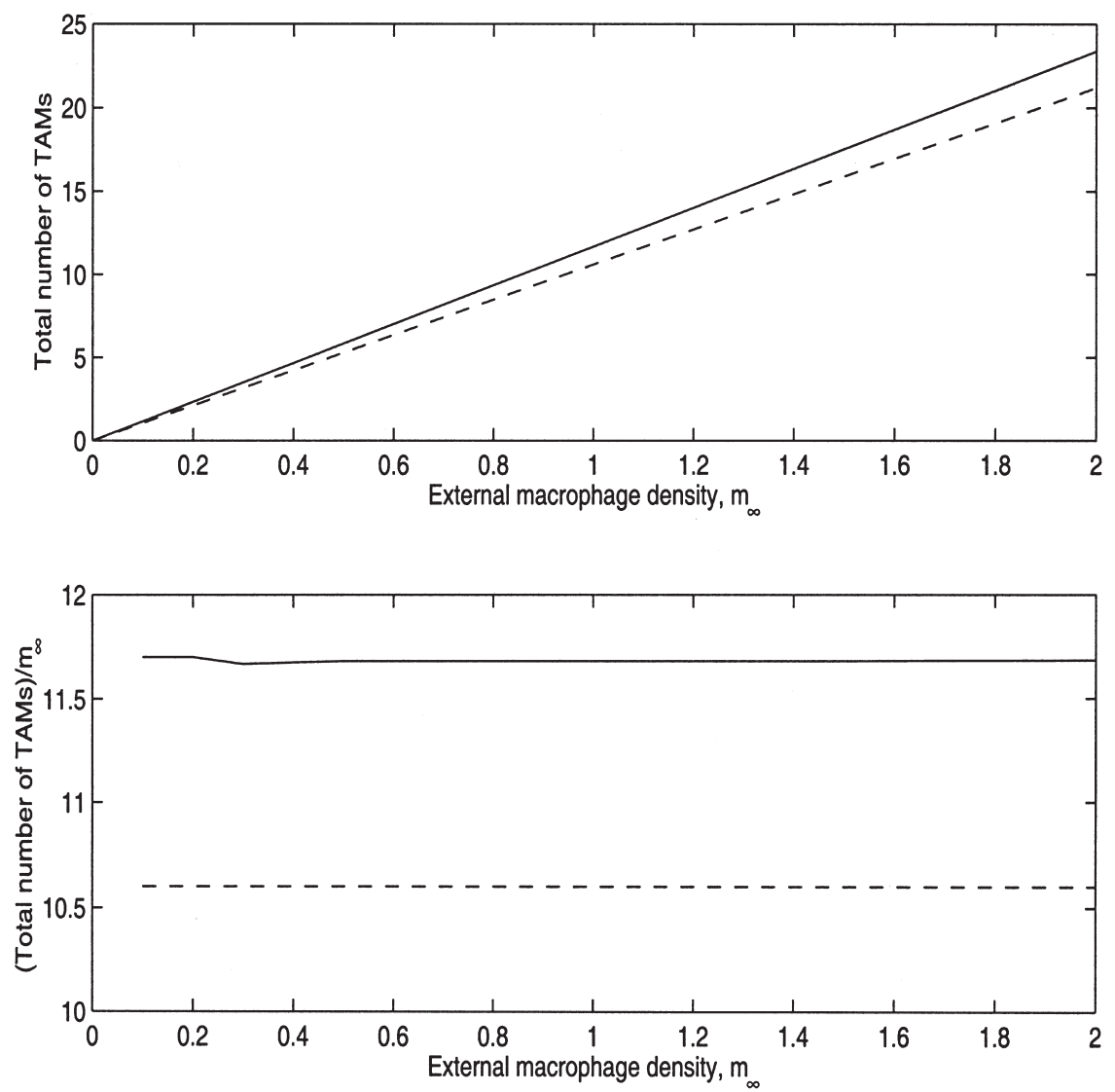

FIGURE 9 In the upper panel we show that $M\left(t=10 ; R, \lambda_{1}\right)$ increases with $m_{\infty}$, the density of macrophages outside the spheroid. Large values of $m_{\infty}$, correspond to still experiments, in which the macrophages settle at the bottom of the well, whereas small values of $m_{\infty}$ correspond to experiments in which the fluid medium is continuously agitated. By plotting $M_{\text {norm }}=M\left(t=10 ; R, \lambda_{1}\right) / m_{\infty}$ against $m_{\infty}$ in the lower panel we show that the total number of infiltrating macrophages increases approximately linearly with $m_{\infty}$. Key: hepa-1 spheroids (solid line), C4 spheroids (dashed line). Parameter values: as per Fig. 4.

Case I. $0<R^{2}<6\left(1-c_{\mathrm{H}}\right)$.

Within small non-hypoxic tumours, the nutrient concentration satisfies $c>c_{\mathrm{H}}$ everywhere. As a result there is no chemoattractant production and

$$
\omega(r) \equiv 0 \quad \forall r \in[0, R] .
$$

In this case, random motion governs macrophage infiltration into both the hepa-1 and $\mathrm{C} 4$ spheroids. Moreover, in the limit as $t \rightarrow \infty$, Eq. (22) reduces to give

$$
0 \sim \frac{1}{r^{2}} \frac{\mathrm{d}}{\mathrm{d} r}\left(\frac{r^{2}\left(c-c_{\mathrm{N}}\right)}{1+c-2 c_{\mathrm{N}}} \frac{\mathrm{d} m}{\mathrm{~d} r}\right)
$$

Integrating twice with respect to $r$ and imposing Eqs. (24) and (27), we deduce that

$$
m \rightarrow m_{\infty}, \quad \forall r \in[0, R] \quad \text { as } t \rightarrow \infty .
$$

Thus, when there is no chemoattractant present, the macrophages distribute themselves uniformly throughout the tumour.

Case II. $\quad 6\left(1-c_{\mathrm{H}}\right)<R^{2}<6\left(1-c_{\mathrm{N}}\right)$.

Within intermediate-sized, non-necrotic tumours, chemoattractant production is localised to hypoxic sites where $c_{\mathrm{N}}<c<c_{\mathrm{H}}$ and Eq. (22) admits the following solution:

$\omega(r)=\left\{\begin{array}{l}\lambda_{1}+\frac{A \sinh r}{r} \text { for } 0<r<R_{\mathrm{H}} \\ \frac{A \sinh r}{r}+\frac{\lambda_{1}}{r}\left[R_{\mathrm{H}} \cosh \left(r-R_{\mathrm{H}}\right)+\sinh \left(r-R_{\mathrm{H}}\right)\right] \\ \quad \text { for } R_{\mathrm{H}}<r<R\end{array}\right.$

where the constant of integration, $A$, is determined by imposing Eq. (24):

$$
\begin{aligned}
& A\left[R \cosh R-\left(1-h_{\omega} R\right) \sinh R\right] \\
& \quad=\lambda_{1}\left[R_{\mathrm{H}}\left(1-h_{\omega} R\right)-R\right] \cosh \left(R-R_{\mathrm{H}}\right)+\lambda_{1}[(1 \\
& \left.\left.\quad-h_{\omega} R\right)-R R_{\mathrm{H}}\right] \sinh \left(R-R_{\mathrm{H}}\right) .
\end{aligned}
$$

With $\omega(r)$ defined by Eq. (30), the long-time behaviour of the macrophages satisfies

$$
0=\frac{1}{r^{2}} \frac{\mathrm{d}}{\mathrm{d} r}\left\{\frac{r^{2}\left(c-c_{\mathrm{N}}\right)}{1+c-2 c_{\mathrm{N}}}\left(\frac{\mathrm{d} m}{\mathrm{~d} r}-m \frac{\mathrm{d} \omega}{\mathrm{d} r}\right)\right\}
$$



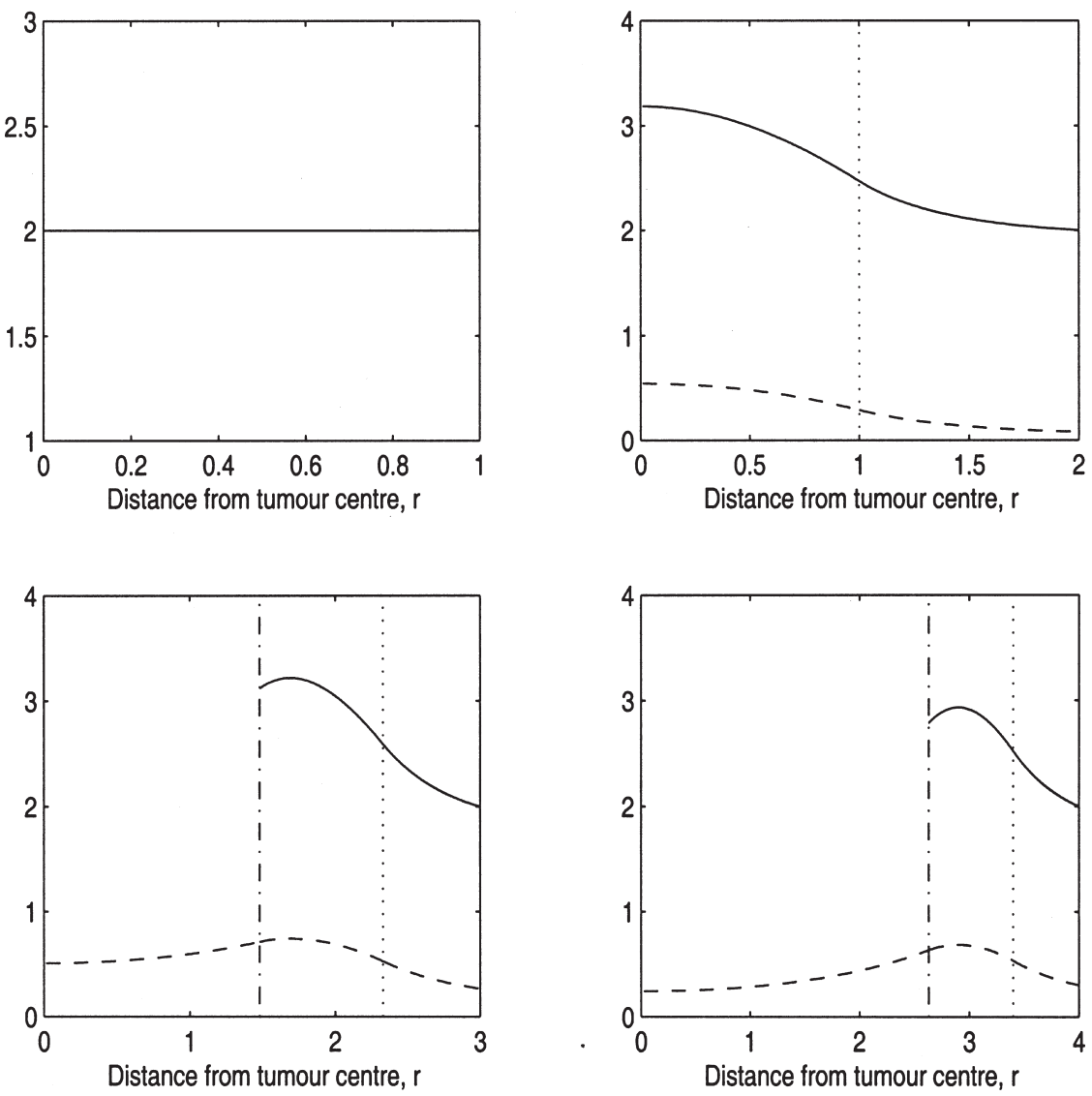

FIGURE 10 Here we show how, for chemoattractant-producing, hepa-1 spheroids, the analytically-constructed, equilibrium chemoattractant and macrophage profiles vary with $R$. The results are in good agreement with numerical solutions of the full model (see Fig. 5). The dotted and dot-dashed vertical lines denote the hypoxic and necrotic boundaries $R_{\mathrm{H}}$ and $R_{\mathrm{N}}$, respectively. Key: chemoattractant concentration (dashed line); macrophage density (solid line). Parameter values: as per Fig. 4.

Integrating once with respect to $r$, and imposing Eqs. (24) and (27), we deduce that

$$
0=\frac{r^{2}\left(c-c_{\mathrm{N}}\right)}{1+c-2 c_{\mathrm{N}}}\left(\frac{\mathrm{d} m}{\mathrm{~d} r}-m \frac{\mathrm{d} \omega}{\mathrm{d} r}\right)=h_{m}\left(m_{\infty}-m(R)\right) .
$$

Since $h_{m} \neq 0$ and $c>c_{N} \forall r \in[0, R]$, we deduce that $m(R)=m_{\infty}$ and

$$
\frac{\mathrm{d} m}{\mathrm{~d} r}=m \frac{\mathrm{d} \omega}{\mathrm{d} r} .
$$

Integrating again with respect to $r$, we deduce that the equilibrium distribution of macrophages within nonnecrotic tumours which possess a hypoxic region is given by

$$
m(r)=m_{\infty} \exp [\omega(r)-\omega(R)] \quad \text { for } r \in[0, R] .
$$

We note that the maximum macrophage density coincides with the maximum chemoattractant concentration, which occurs at $r=0$. We note also that for chemoattractant-deficient spheroids $\left(\lambda_{1}=0\right) \omega(r) \equiv 0$ and, hence, $m(r)=m_{\infty}$ for $r \in\left[R_{\mathrm{H}}, R\right]$. Thus we predict that within hepa-1 spheroids the macrophages will accumulate at the centre of the tumour whereas within
C4 spheroids the macrophages will distribute themselves throughout the tumour volume.

Case III. $6\left(1-c_{\mathrm{N}}\right)<R^{2}$.

Within well-developed tumours that possess hypoxic and necrotic regions, the chemoattractant profile is given by:

$$
\omega=\left\{\begin{aligned}
& \frac{\tilde{A} \sinh r}{r} \text { for } 0<r<R_{\mathrm{N}} \\
& \lambda_{1}+\frac{\bar{A} \sinh r}{r}-\frac{\lambda_{1}}{r}\left[R_{\mathrm{N}} \cosh \left(r-R_{\mathrm{N}}\right)+\sinh \left(r-R_{\mathrm{N}}\right)\right] \\
& \quad \text { for } R_{\mathrm{N}}<r<R_{\mathrm{H}} \\
& \omega_{P}(r) \text { for } R_{\mathrm{H}}< \quad r<R
\end{aligned}\right.
$$

where

$$
\begin{aligned}
\omega_{P}(r)= & \frac{\tilde{A} \sinh r}{r}-\frac{\lambda_{1}}{r}\left[R_{\mathrm{N}} \cosh \left(r-R_{\mathrm{N}}\right)+\sinh \left(r-R_{\mathrm{N}}\right)\right] \\
& +\frac{\lambda_{1}}{r}\left[R_{\mathrm{H}} \cosh \left(r-R_{\mathrm{H}}\right)+\sinh \left(r-R_{\mathrm{H}}\right)\right]
\end{aligned}
$$


and the constant of integration $\tilde{A}$ is determined by imposing the flux boundary condition at $r=R$ :

$$
\begin{aligned}
\tilde{A}[R & \left.\cosh R-\left(1-h_{\omega} R\right) \sinh R\right] \\
= & \lambda_{1}\left[R_{\mathrm{H}}\left(1-h_{\omega} R\right)-R\right] \cosh \left(R-R_{\mathrm{H}}\right)+\lambda_{1}\left[\left(1-h_{\omega} R\right)\right. \\
& \left.-R R_{H}\right] \sinh \left(R-R_{\mathrm{H}}\right)-\lambda_{1}\left[R_{\mathrm{N}}\left(1-h_{\omega} R\right)\right. \\
& -R] \cosh \left(R-R_{\mathrm{N}}\right)-\lambda_{1}\left[\left(1-h_{\omega} R\right)-R R_{\mathrm{N}}\right] \sinh (R \\
& \left.-R_{\mathrm{N}}\right) .
\end{aligned}
$$

Using an argument similar to that used to determine the macrophage distribution for Case II, it is possible to show that $m(R)=m_{\infty}$ and

$$
m(r)=m_{\infty} \exp [\omega(r)-\omega(R)] .
$$

In Fig. 10 we summarise the analytical results derived above, by plotting the steady-state chemoattractant concentration $\omega$ and the macrophage density $m$ for chemoattractant-producing (hepa-1) spheroids of varying sizes. Whilst the qualitative forms of the profiles for the different spheroids are in good agreement with those obtained by solving the original, time-dependent model (see Fig. 5), we note that in each case the mean macrophage density, as defined by Eq. (29), is higher for the steady-state profiles than for the time-dependent solutions, provided the tumour has developed a hypoxic regions.

Recall that at equilibrium the macrophage density on the tumour boundary is given by $m(R, t)=m_{\infty}$. Comparing Figs. 5 and 10, we conclude that the partialdifferential-equation (PDE) model has not reached its steady state at the time chosen $(t=10$ in dimensionless units). We repeated the numerical simulations of the PDE model, extending the period of simulation from $t=10$ to $t=100$. Even at this later time (results not shown), the equilibrium profiles had not been attained. Thus we conclude that whilst the analytically-constructed steady state solutions provide some insight into the qualitative form of the chemoattractant concentration and macrophage density, the time taken to realise the steady states is too long to be biologically relevant.

\section{DISCUSSION}

In this paper we have presented a mathematical model that describes macrophage infiltration into avascular tumours or spheroids via a combination of random motion and chemotaxis. A combination of numerical and analytical results were used to show how the tumour's size (and spatial structure) and the ability of its constituent cells to produce chemoattractants influence the distribution patterns of the macrophages.

Numerical solutions of the model equations were compared with experiments in which the infiltration patterns of macrophages into two types of tumour spheroids were studied (Leek, 1999). The spheroids were cultured from hepa-1 cells, which are believed to express a chemoattractant under hypoxia, and $\mathrm{C} 4$ cells, which do not. By comparing the numerically-constructed solutions with the experimental data we were able to generate a number of predictions which are consistent with Leek's results and which we summarise below.

- Observed differences in macrophage infiltration patterns may be due to the hepa- 1 spheroids producing chemoattractant at a higher rate than $\mathrm{C} 4$ spheroids.

- Neither hypoxia nor necrosis are necessary for macrophage infiltration: macrophages can migrate towards and within tumours by random motion alone.

- The number of infiltrating macrophages increases with tumour size for both the chemoattractant-producing and chemoattractant-deficient spheroids.

There are also a number of discrepancies between the numerical simulations and the experimental data that merit discussion. For example, the numerical simulations predict that at all times the hepa- 1 spheroids recruit larger numbers of macrophages than $\mathrm{C} 4$ spheroids of the same size (see Figs. 7 and 8). This is consistent with Leek's experimental observations at early times but does not explain the marked increase in macrophage infiltration into $\mathrm{C} 4$ spheroids that occurs after $12 \mathrm{~h}$ co-culture (see Figs. 2 and 3). There are at least two possible explanations for this discrepancy. Referring to Table I we note that the detailed spatial structures of the hepa-1 and $\mathrm{C} 4$ spheroids are quite different, with the hepa-1 spheroids possessing considerably larger regions of necrosis than the $\mathrm{C} 4$ spheroids. This suggests that the kinetics of the two cell lines are also different and, in particular, that the $\mathrm{C} 4$ spheroids consume oxygen at a lower rate than the hepa-1 spheroids. Such a difference would delay the onset of hypoxia and necrosis within the $\mathrm{C} 4$ spheroids relative to the hepa-1 spheroids and would result in the mean macrophage density in Fig. 8 maintaining its maximum value (that attained prior to necrosis) for a larger range of spheroid sizes. Such a shift in the mean macrophage density curve may be sufficient to explain Leek's observations. Alternatively, the absence of a functioning hif-1 gene in C4 tumour cells may lead to the induction of other signalling pathways when the cells are subject to prolonged periods of hypoxic stress (e.g. upregulation of the production of macrophage inhibitory factor which immobilises macrophages within hypoxic regions).

Comparing Figs. 3 and 5 we note that the qualitative form of the macrophage infiltration patterns for the (experimentally-observed) $\mathrm{C} 4$ spheroids and the (numerically-simulated) chemoattractant-deficient spheroids are different, with the maximum macrophage density occurring on the tumour boundary for the numerical simulations and inside the tumour for the experimental data, as for the chemoattractant-producing spheroids. 
Guided by this difference we predict that cells within the C4 spheroids are producing macrophage chemoattractants, albeit at a lower rate than the hepa-1 spheroids.

There are many ways in which our mathematical model could be extended and improved. In the existing model the pro- and anti-tumour functions that macrophages may perform have been neglected, and the macrophages assumed simply to migrate into the spheroids. Thus a natural extension of the current model would be to include macrophage functions such as tumour cell lysis and the production by the macrophages of additional macrophage chemoattractants (Owen and Sherratt, 1997; 1998; 1999). Other natural extensions would be to allow for growth of the tumour during macrophage infiltration and to distinguish between the volumes occupied by different cell types (e.g. tumour cells and macrophages) within the spheroid. These modifications could be achieved by adopting the modelling framework proposed by Ward and King (1997). We would then be able to predict whether the increase in spheroid volume observed by Leek was due to swelling caused by the infiltrating macrophages or to tumour cell proliferation. We would also be able to investigate whether the infiltrating macrophages displace tumour cells originally located at the necrotic boundary and, if this is the case, whether it diminishes the strength of the chemoattractant signal that recruits additional macrophages into the spheroid. By further extending the model to include multiple macrophage populations (e.g. normal and genetically-engineered macrophages), with each population carrying out different functions, it should be possible to test the feasibility of using geneticallyengineered macrophages to target hypoxic sites within solid tumours, the modified macrophages being designed to release anti-angiogenic or cytotoxic chemicals under hypoxia.

Given the long-term aim of using geneticallyengineered macrophages to target hypoxic tumour sites, we anticipate that higher densities of infiltrating macrophages will lead to greater rates of tumour cell lysis. For this reason it is important to be able to estimate the mean density of macrophages within a particular tumour and to see how this value is affected by the tumour's size, spatial structure and whether the tumour cells are producing macrophage chemoattractants. In Fig. 8 we showed how the mean macrophage density, at a fixed time-point, varied with tumour size for chemoattractantproducing tumour cells and chemoattractant-deficient cells. In the latter case, the mean macrophage density decreased steadily as the tumour increased in size. A similar pattern was observed for the chemoattractantproducing spheroids, except for a small rise in the mean macrophage density, which coincided with the appearance of a hypoxic region. These results suggest that the delivery of genetically engineered macrophages to tumours will be strongly dependent on not only the size and spatial structure of the tumours but also on the rate at which the constituent cells produce macrophage chemoattractants under hypoxia.
In conclusion, whilst our model of macrophage infiltration is undoubtedly simplistic, it represents a good starting point for future work that may help in the search for effective treatments to manage cancer growth.

\section{References}

Adam, J.A. (1987) "A mathematical model of tumour growth II. Effects of geometry and spatial uniformity on stability", Math. Biosci. 86, $183-211$.

Babaev, V.R., Fazio, S., Gleaves, L.A., Carter, K.J., Semenkovich, C.F. and Linton, M.F. (1999) "Macrophage lipoprotein lipase promotes foam cell formation and atherosclerosis in vivo", J. Clin. Invest. 103, $1697-1705$.

Bennet, N.T. and Schulz, G.S. (1993) "Growth factors and wound healing: part II. role in normal and chronic wound healing", Am. J. Surg. 166, 74-81.

Bischof, R.J., Zafiropoulos, D., Hamilton, J.A. and Campbell, I.K. (2000) "Exacerbation of acute inflammatory arthritis by the colonystimulating factors CSF-1 and granulocyte macrophage (GM)-CSF: evidence of macrophage infiltration and local proliferation", Clin. Exp. Imm. 119, 361-367.

Bottazzi, B., Polentarutti, N., Acero, R., Balsari, A., Boraschi, D., Ghezzi, P., Salmona, M. and Mantovani, A. (1983) "Regulation of the macrophage content of neoplasms by chemoattractants", Science $\mathbf{2 2 0}$, 210-212.

Bottazzi, B., Ghezzi, P., Taraboletti, G., Salmona, M., Colombo, N., Bonnazzi, C., Mangioni, C. and Mantovani, A. (1985) "Tumourderived chemotactic factor(s) from human ovarian carcinoma: evidence for a role in the regulation of macrophage content of neoplastic tissues", Int. J. Cancer 36, 167-173.

Dupe, V., Chyselinck, N.B., Thomazy, V., Nagy, L., Davies, P.J.A., Chainbon, P. and Mark, M. (1999) "Essential roles of retinoic acid signalling in interdigital apoptosis and control of BMP-7 expression in mouse autopods", Dev. Biol. 208, 30-43.

Elgert, K.D., Alleva, D.G. and Mullins, D.W. (1998) "Tumour-induced immense dysfunction: the macrophage connection", J. Leuk. Biol. 64, 275-290.

Griffiths, L., Binley, K., Iqball, S., Kan, O., Maxwell, P., Ratcliffe, P., Lewis, C.E., Harris, A.L., Kingsman, S. and Naylor, S. (2000) "The macrophage - a novel system to deliver gene therapy to pathological hypoxia", Gene Ther. 7, 255-262.

Gordon, S. (1995) "The macrophage”, BioEssays 17, 977-986.

Greenspan, H.P. (1972) "Models for the growth of a solid tumour by diffusion", Stud. Appl. Math. 52, 317-340.

Koong, A.C., Denko, N.C., Hudson, K.M., Schindler, C., Swiersz, L., Koch, C., Evans, S., Ibrahim, H., Le, Q.T., Terris, D.J. and Giaccia, A.J. (2000) "Candidate genes for the hypoxic tumor phenotype", Cancer Res. 60, 883-887.

Leek, R.D. (1999) The Role of Tumour Associated Macrophages in Breast Cancer Angiogenesis PhD Thesis Oxford Brookes University (Oxford).

Leek, R.D., Lewis, C.E., Whitehouse, R., Greenall, M., Clarke, J. and Harris, A.L. (1996) "Association of macrophage infiltration with angiogenesis and prognosis in invasive breast carcinoma", Cancer Res. 56, 4625-4629.

Leek, R.D., Lewis, C.E. and Harris, A.L. (1997) "The role of macrophages in tumour angiogenesis", In: Bicknell, R., Lewis, C.E. and Ferrara, N., eds, Tumour Angiogenesis.

Leek, R.D., Landers, R.J., Harris, A.L. and Lewis, C.E. (1999) "Necrosis correlates with high vascular density and focal macrophage infiltration in invasive carcinoma of the breast", $\mathrm{Br}$. J. Cancer 79, 991-995.

Leek, R.D., Hunt, N.C., Landers, R.J., Lewis, C.E., Royds, J.A. and Harris, A.L. (2000) "Macrophage infiltration is associated with VEGF and EGFR expression in breast cancer", J. Pathol. 190, 430-436.

Lewis, C.E., Leek, R., Harris, A. and McGee, J.O.D. (1995) "Cytokine regulation of angiogenesis in breast cancer-the role of tumourassociated macrophages", J. Leuk. Biol. 57, 747-751.

Lewis, J.S., Lee, J.A., Underwood, J.C.E., Harris, A.L. and Lewis, C.E. (1999) "Macrophage responses to hypoxia, relevance to disease mechanisms", J. Leuk. Biol. 66, 889-900. 
Lewis, J.S., Landers, R.J., Underwood, J.C.E., Harris, A.L. and Lewis, C.E. (2001) "Expression of VEGF by macrophages is upregulated in avascular areas of breast carcinomas", J. Pathol. 192, 150-158.

Mantovani, A., Botazzi, B., Colotta, F., Sozzani, S. and Ruco, L. (1992) "The origin and function of tumour-associated macrophages", Immunol. Today 13, 265-270.

Mapp, P.I., Grootvold, M.C. and Blake, D.R. (1995) "Hypoxia, oxidative stress and rheumatoid arthritis", Br. Med. Bull. 51, 419-436.

Negus, P.M., Stamp, G.W., Hadley, J. and Balkwill, F.R. (1997) "Quantitative assessment of the leukocyte infiltrate in ovarian cancer and its relationship to the expression of $\mathrm{C}-\mathrm{C}$ chemokines", $\mathrm{Am}$. J. Pathol. 150, 1723-1734.

Negus, P.M., Turner, L., Burke, F. and Balkwill, F.R. (1998) "Hypoxia down-regulates MCP-1 expression: implications for macrophage distribution in tumours", J. Leuk. Biol. 63, 758-765.

Owen, M.R. and Sherratt, J.A. (1997) "Pattern formation and spatiotemporal irregularity in a model for macrophage-tumour interactions", J. Theor. Biol. 189, 63-80.

Owen, M.R. and Sherratt, J.A. (1998) "Modelling the macrophage invasion of tumours: effects on growth and composition", IMA J. Math. Appi. Med. Biol. 15, 165-185.

Owen, M.R. and Sherratt, J.A. (1999) "Mathematical modelling of macrophage dynamics in tumours", Math. Mod. Meth. Appl. Sci. 9, $513-539$.

Pupa, S.M., Bufalino, R., Invernizzi, A.M., Andreola, S., Rilke, F., Lombardi, L., Colnaghi, M.I. and Menard, S. (1996) "Macrophage infiltrate and prognosis in c-erbB-2-overexpressing breast carcinomas", J. Clin. Oncol. 14, 85-94.

Santilli, S.M., Fiegel, V.D., Aldridge, D.E. and Knighton, D.R.Q. (1991) "Rabbit aortic endothelial cell hypoxia induces secretion of transforming growth factor beta and augments macrophage, adhesion in vitro", Ann. Vasc. Surg. 5, 429-438.

Shi, X., Le, J.L., Abbruzzese, B., Wang, N., Mujaida, K., Matsushima, S., Huang, Q., Xiong, Q. and Xic, K.J. (1999) "Cooperation between transcription factor AP-1 and NF-kappa B in the induction of interleukin-8 in human pancreatic adenocarcinoma cells by hypoxia", Interferon Cytokine Res. 19, 1363-1371.

Toomey, D., Harmey, J., Condron, C., Kay, E. and Bouchier-Hayes, D. (1999) "Phenotyping of immune cell infiltrates in breast and colorectal tumours", Immunol. Invest. 28, 29-41.

Tran, C.L., Jones, A.D., Cullen, R.T. and Donaldson, K. (1999a) "Mathematical modelling of the retention and clearance of lowtoxicity particles in the lung", Inhal. Toxicol. 11, 1059-1076.

Tran, C.L., Jones, A.D., Cullen, R.T. and Donaldson, K. (1999b) "Exploration of the mechanisms of retention and clearance of lowtoxicity particles in the rat lung using a mathematical model", Inhal. Toxicol. 11, 1077-1108

Ward, J.P. and King, J.R. (1997) "Mathematical modelling of avascular tumour growth", IMA J. Math. Appl. Med. Biol. 14, 39-69.

Wodarz, D., Lloyd, A.L., Jansen, V.A.A. and Nowak, M.A. (1998) "Dynamics of macrophage and T cell infection by HIV", J. Theor. Biol. 196, 101-113. 


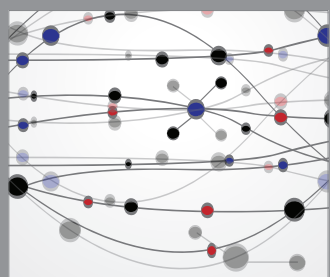

The Scientific World Journal
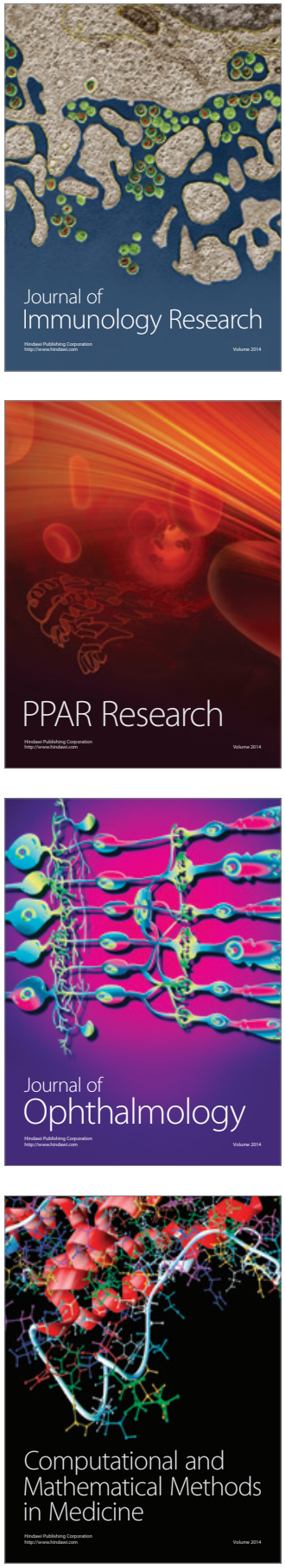

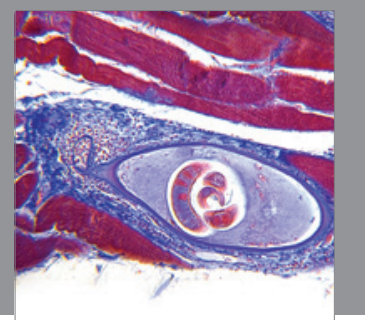

Gastroenterology

Research and Practice
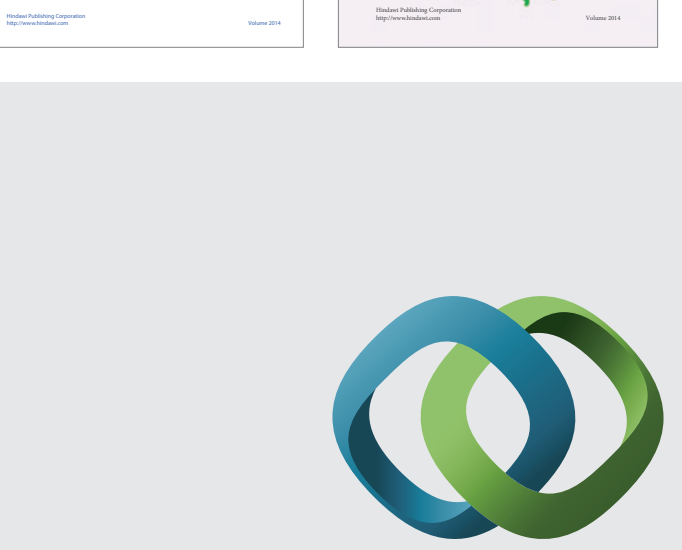

\section{Hindawi}

Submit your manuscripts at

http://www.hindawi.com
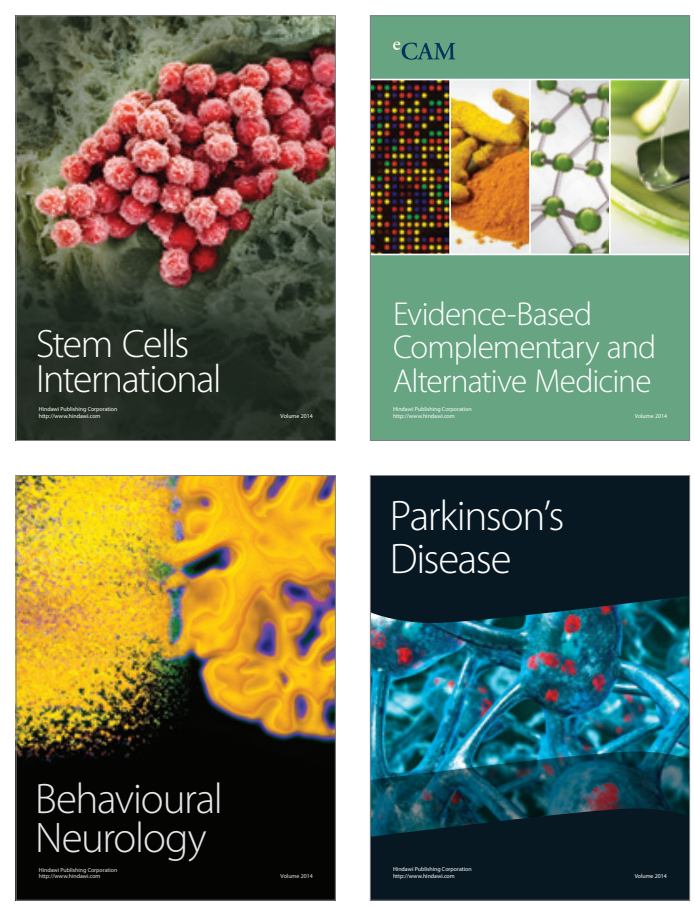

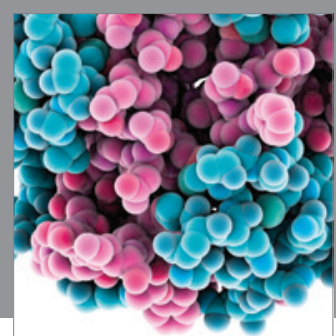

Journal of
Diabetes Research

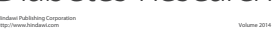

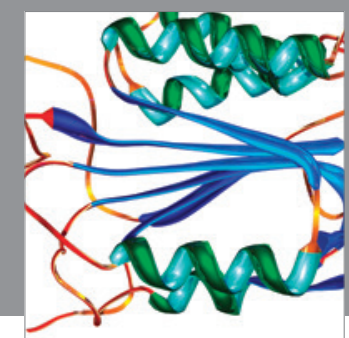

Disease Markers
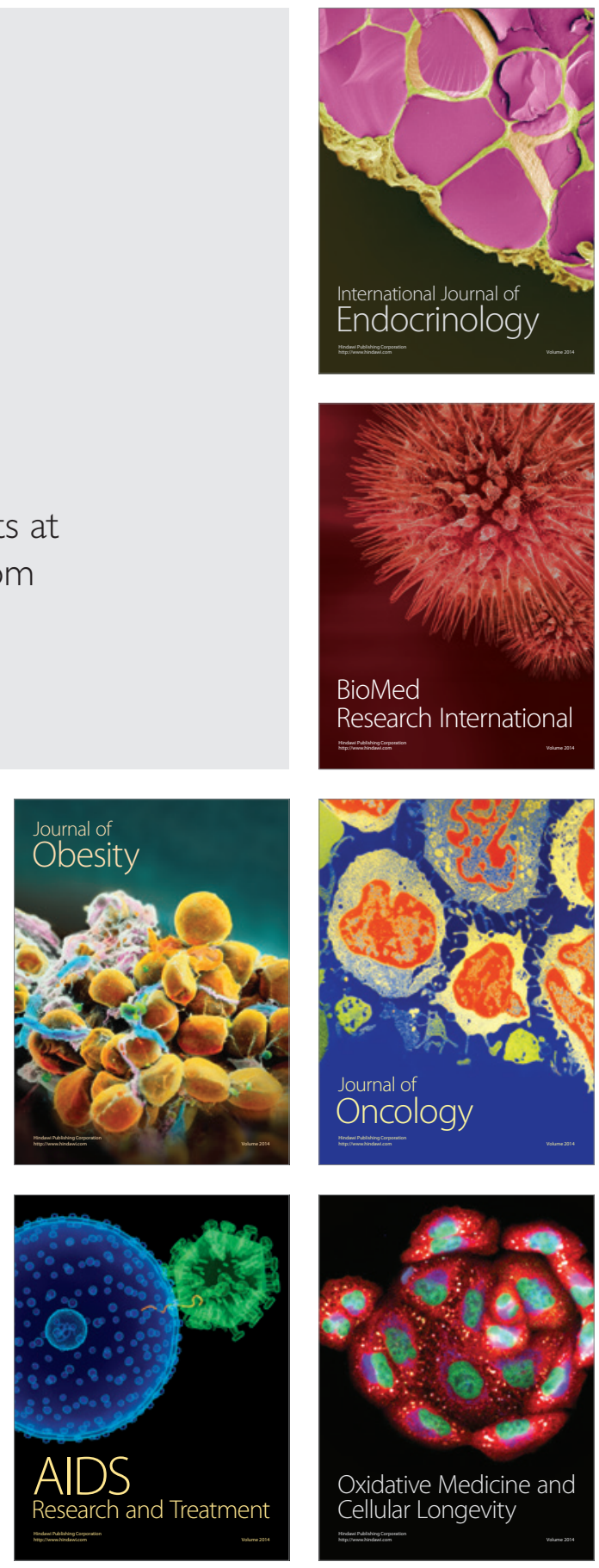